\title{
Oscillations and evolution of a hot and dense gas of flavor neutrinos: a quantum field theory study.
}

\author{
D. Boyanovsky, ${ }^{1, \text { A }}$ and C.M. Ho ${ }^{1, \text { 由 }}$ \\ ${ }^{1}$ Department of Physics and Astronomy, University of Pittsburgh, Pittsburgh, Pennsylvania 15260, USA
}

(Dated: November 11, 2018)

\begin{abstract}
We study the time evolution of the distribution functions for hot and or degenerate gases of two flavors of Dirac neutrinos as a result of flavor mixing and dephasing. This is achieved by obtaining the time evolution of the flavor density matrix directly from quantum field theory at finite temperature and density. The time evolution features a rich hierarchy of scales which are widely separated in the nearly degenerate or relativistic cases and originate in interference phenomena between particle and antiparticle states. In the degenerate case the flavor asymmetry $\Delta N(t)$ relaxes to the asymptotic limit $\Delta N(\infty)=\Delta N(0) \cos ^{2}(2 \theta)$ via dephasing resulting from the oscillations between flavor modes that are not Pauli blocked, with a power law $1 / t$ for $t>t_{s} \approx 2 k_{F} / \Delta M^{2} . k_{F}$ is the largest of the Fermi momenta. The distribution function for flavor neutrinos and antineutrinos as well as off-diagonal densities are obtained. Flavor particle-antiparticle pairs are produced by mixing and oscillations with typical momentum $k \sim \bar{M}$ the average mass of the neutrinos. An effective field theory description emerges on long time scales in which the Heisenberg operators obey a Bloch-type equation of motion valid in the relativistic and nearly degenerate cases. We find the non-equilibrium propagators and correlation functions in this effective theory and discuss its regime of validity as well as the potential corrections.
\end{abstract}

PACS numbers: 14.60.Pq,12.15.Ff,11.90.+t

\section{INTRODUCTION}

Neutrinos are the bridge between particle physics, astrophysics, cosmology and nuclear physics 1, 2, 3, 4, 5, 6], and after almost four decades of the prescient suggestion that neutrinos may oscillate 7, 81, a wealth of experimental data confirms that neutrinos are massive and that different flavors mix and oscillate $9,10,11,12$, 13]. Neutrino masses and mixing decidedly points to new physics beyond the standard model and profoundly impacts on the physics, astrophysics and cosmology of neutrinos. Neutrino oscillations in matter may provide an explanation of the solar neutrino problem by the resonant conversion of flavor neutrinos in the medium, namely the MSW effect [16, 17] (for recent reviews see [1]-15]). The dynamical aspects of neutrino oscillations in extreme conditions of temperature and density play an important role in Big Bang Nucleosynthesis (BBN) and in the lepton asymmetry in the early Universe [18] (for a recent review see [6]) as well as in the physics of core collapse supernovae and the formation, evolution and cooling of neutron stars 19, 20, 21]. The study of the dynamical evolution of a hot and/or dense gas of neutrinos that include mixing as well as collisions has been and continues to be the subject of much attention in the literature. Neutrino mixing and oscillations introduce a novel aspect in the description of flavor equilibration, since the weak interactions involve flavor (weak) eigenstates while time evolution is described in terms of mass eigenstates. Therefore in a dense and/or hot medium where neutrino interactions cannot be neglected collisional processes must be studied on the same footing as the dynamics of oscillations. Furthermore in a dense background of neutrinos such as is the case in the early Universe during the time relevant for BBN or during the time scale of neutrino trapping in a protoneutron star, the neutral current interaction leads to a contribution to the neutrino self-energy from forward scattering off the neutrino background akin to the contribution from the electron plasma that leads to MSW resonance enhancement [16. In dense neutrino gases, this self-energy contribution leads to a non-linear problem for the evolution of a given neutrino interacting with the neutrino background.

The dynamics of neutrino oscillations was originally studied in terms of Bloch-type equations akin to the equation of motion for a spin in a magnetic field [1, 2, 3, 16, 22] which are generally valid for single particle descriptions in the relativistic limit. For the case of single particle states this equation of motion for neutrino oscillations was derived from the underlying field theory in the relativistic limit [5, 23. This formulation of the dynamics of oscillations of single particle states was extended to a kinetic description of oscillations and mixing in a medium [24, 25, 26]. The

*Electronic address: boyan@pitt.edu

†Electronic address: cmho@phyast.pitt.edu 
resulting equations in principle include the effects of collisions as well as the non-linearities arising from neutrino forward scattering off a neutrino background. They have been implemented to study the evolution of the neutrino distribution functions in the early Universe 6 , 27, 28, 29, 30, 31], in supernovae [32, 33, 34] as well as to study the relic neutrino asymmetry [35]. Novel fascinating self-synchronization phenomena emerges as a consequence of the non-linearities in a neutrino background with potential implications on CP (and baryon) asymmetry in the early Universe [6, 28].

An alternative quantum field theory treatment of neutrinos in the medium used the ingredients of thermal field theory [36, 37] combined with a self-consistent treatment in the case of a neutrino background 38]. Since the main method in this approach relies on the equilibrium description of thermal field theory, there is an underlying assumption that the neutrino background is nearly in equilibrium.

More recently the validity of the single particle picture that underlies the kinetic equations for neutrinos in a medium has been critically re-examined [39].

In our view, the study of neutrino oscillations and mixing in the case of a dense and or hot neutrino background either via the set of kinetic equations [24, 25, 26] or the thermal field theory approach invoke a variety of approximations some of which are not very clear. In the kinetic description several approximations are involved, from neglecting interference terms between particles and antiparticles by restricting the Hamiltonian 24] to some time averaging and restriction to single particle evolution [26]. Some of these approximations motivated the study of ref. [39].

A full quantum field theory treatment of neutrino mixing reveals a more complex picture of oscillations beyond that of the single particle description [40, 41, 42]. The authors of these references pointed out that a careful treatment of the Fock representation of flavor states leads to novel contributions to the oscillation formula even for single particle states. While it has been argued recently that Fock states of flavor neutrinos may not be relevant for S-matrix processes [43] a quantum statistical mechanics of dense and or hot flavor neutrino gases must necessarily rely on the Fock representation (occupation number) for flavor neutrino states.

A quantum statistical description of a dense and or hot gas of flavor neutrinos requires the notion of an occupation number which inevitably implies a description in terms of Fock flavor states. Furthermore a chemical potential associated with a flavor neutrino is a variable conjugate to the number of these flavor neutrinos.

Regardless of whether the variety of approximations usually invoked are justified for practical purposes, the study of the dynamics of neutrino mixing and oscillations from the point of view of quantum field theory is clearly of fundamental importance as a prelude towards physics beyond the standard model. While there have been studies of the quantum field theory aspects in vacuum we are not aware of any previous study of the quantum field theory of mixing in a dense and or hot medium with neutrinos.

\section{The goal of this study:}

In the presence of flavor mixing, individual flavor number is not conserved and a density matrix that is diagonal in the flavor Fock basis will evolve in time and develop off diagonal elements.

Hence time evolution of a dense or hot neutrino gas has to be studied as a quantum mechanical initial value problem: an initial density matrix which is diagonal in the flavor basis is evolved in time with the full Hamiltonian with flavor mixing. In this article we focus on studying precisely the time evolution of a dense or hot flavor neutrino gas in the simplest case of free field theory. Our goal is to study the evolution of an initially prepared density matrix which is diagonal in the flavor basis and describes a quantum gas of flavor neutrinos at finite density or finite temperature. We undertake the study of the dynamics in free field theory as a prelude towards a complete understanding of oscillation phenomena in weak interactions. The first step of any systematic program must be the understanding at the simplest level. As will be detailed below, studying the dynamics of oscillations and mixing in a dense and/or hot medium even at the level of free field theory reveals a wealth of subtle and important phenomena which leads to a firmer understanding of the validity of the various approximations as well as highlighting the potential corrections.

The problem that we study can be stated succinctly as follows ${ }^{1}$ : Consider that at a given initial time we have a "box" that contains a hot or dense gas of flavor neutrinos with a given single particle distribution consistent with Fermi-Dirac statistics, how does this ensemble evolve in time?, how do the populations of flavor neutrinos evolve in time?, how do flavor neutrinos propagate in the medium??.

While our ultimate goal is to study the evolution in the presence of the weak interactions, we begin our study in this simplest free field theory case and the case of two flavors with the following goals in mind

- To study the evolution directly from the underlying quantum field theory without making any approximations. This study will clarify the nature of the various approximations invoked in the literature and exhibit the potential corrections.

\footnotetext{
${ }^{1}$ D.B. thanks S. Reddy for stating the question over dinner.
} 
- By keeping the full evolution, the different time scales will emerge thus paving the way to providing a firmer understanding of coherence effects as well as the time averaging implied by several approximations.

- A first principle derivation of kinetic equations and or Boltzmann equations require the propagators for the fields 44] in the medium. Thus the study of the evolution in free field theory is the starting point for a systematic treatment of oscillations and collisions in a medium with a neutrino background.

- As it will become clear below, the study of even the simple free field theory case reveals a wealth of phenomena as a consequence of flavor mixing, which to the best of our knowledge has not been recognized and explored fully before in the case of finite temperature and density. The full quantum field theory treatment unambiguously reveals all the complexities associated with flavor mixing and allows a systematic implementation of several approximations which clarify the regime of validity of the single particle description and provide an understanding of the corrections.

Brief summary of the results: Our main results are briefly summarized as follows,

- The dynamics of neutrino oscillations of a dense and or hot gas of flavor neutrinos features a hierarchy of time scales. The fast time scales are associated with interference effects between particle and antiparticle states while the slow scales emerge from interference between particle states (or antiparticle states) of different masses. In the nearly degenerate or relativistic case the scales are widely separated and processes which involve interference between particle and antiparticle states become subdominant on the slow dynamics.

- An initial flavor asymmetry relaxes towards an asymptotic value $\Delta N(\infty)=\Delta N(0) \cos ^{2}(2 \theta)$ (with $\theta$ being the mixing angle) with a power law $\propto 1 / t$ as a consequence of dephasing. Pauli blocking manifests in that neutrinos of one flavor can only oscillate into un-occupied states of neutrinos of different flavor and dephasing is a consequence of oscillations between Pauli unblocked flavor states. We obtain the explicit time evolution of the distribution functions as well as off-diagonal correlation functions. We discuss the phenomenon of flavor pair production by mixing and oscillations. This is a consequence of the overlap between particle and antiparticle states and results in the production of pairs of flavored neutrinos with typical momenta $k \sim \bar{M}$, the average mass of the neutrinos.

- In the nearly degenerate case (as suggested by the recent combined observations) or in the relativistic case as is likely to prevail in the early universe as well as in core collapse supernovae, the different time scales are widely separated. This allows to establish an "effective" (free) field theory description valid on the slow time scales. The equations of motion for Heisenberg operators in this effective description are the oft quoted Blochtype equations, but the effective field theory also describes the quantum fields. This effective theory allows to construct the Feynman propagators which feature distinct non-equilibrium aspects and to clearly identify the potential corrections and is valid both in the relativistic as well as in the nearly degenerate case.

Our study is organized as follows: in section II the theory corresponding to two flavors of neutrinos as well as the density matrix that describes an initial state of flavor neutrinos is presented. In this section we address the quantization aspects and point out the source of subtle mixing phenomena between particles and antiparticles, confirming previous results in the literature [40]. In sections III and IV we study the evolution of the flavor asymmetry as well as that of the individual distribution functions focusing on the emergence of a hierarchy of scales and extracting the asymptotic long time dynamics as well as the phenomenon of flavor pair production via oscillations. In section $\mathrm{V}$ we present the "effective" field theory that describes the long-time dynamics and discuss its regime of validity. In this section we obtain the Feynman propagators and discuss their non-equilibrium aspects. In section VI we discuss the regime of validity of the several approximations as well as caveats in the formulation and present our conclusions.

\section{NEUTRINO MIXING AND FLAVOR DENSITY MATRIX}

We focus our attention on the evolution of Dirac neutrinos postponing the case of Majorana neutrinos for further discussion elsewhere. Furthermore, we restrict the discussion to the case of two flavors which provides the simplest scenario. Most of the results can be extrapolated to the case of three active flavors including the case of sterile neutrinos, but for the subtleties associated with $\mathrm{CP}$ violating phases which of course are of great interest but will not be addressed here. We will call the flavors the electron and muon neutrino, but the results apply more broadly to active-sterile oscillations. 
Consider the Dirac neutrino fields with the Lagrangian density given by

$$
\mathcal{L}=\bar{\nu}_{e}(x)(i \not \partial) \nu_{e}(x)+\bar{\nu}_{\mu}(x)(i \not \partial) \nu_{\mu}(x)+\left(\begin{array}{cc}
\bar{\nu}_{e}(x) & \bar{\nu}_{\mu}(x)
\end{array}\right)\left(\begin{array}{cc}
m_{e} & m_{e \mu} \\
m_{e \mu} & m_{\mu}
\end{array}\right)\left(\begin{array}{c}
\nu_{e}(x) \\
\nu_{\mu}(x)
\end{array}\right)
$$

where $m_{e \mu}$ is the mixing and we have absorbed a potential phase into a field redefinition. The mass matrix can be diagonalized by introducing a rotation matrix such that

$$
\left(\begin{array}{c}
\nu_{e}(x) \\
\nu_{\mu}(x)
\end{array}\right)=\left(\begin{array}{cc}
C & S \\
-S & C
\end{array}\right)\left(\begin{array}{c}
\psi_{1}(x) \\
\psi_{2}(x)
\end{array}\right)
$$

where for simplicity of notation we defined

$$
C \equiv \cos \theta ; S \equiv \sin \theta
$$

where $\theta$ is the mixing angle. The diagonalized mass matrix then reads

$$
\left(\begin{array}{cc}
M_{1} & 0 \\
0 & M_{2}
\end{array}\right)=\left(\begin{array}{cc}
C & -S \\
S & C
\end{array}\right)\left(\begin{array}{cc}
m_{e} & m_{e \mu} \\
m_{e \mu} & m_{\mu}
\end{array}\right)\left(\begin{array}{cc}
C & S \\
-S & C
\end{array}\right)
$$

In the mass eigenstate basis, the Lagrangian density becomes

$$
\mathcal{L}=\bar{\psi}_{1}(x)\left(i \not \partial-M_{1}\right) \psi_{1}(x)+\bar{\psi}_{2}(x)\left(i \not \partial-M_{2}\right) \psi_{2}(x) .
$$

In what follows, we reserve the latin label $i=1,2$ for the fields associated with the mass eigenstates $\psi$ and the greek label $\alpha=e, \mu$ for the fields associated with the flavor eigenstates $\nu$.

Upon quantization in a volume $V$, the flavor field operators $\nu_{\alpha}(x)$ at time $t=0$ are written as

$$
\begin{aligned}
& \nu_{\alpha}(\vec{x})=\frac{1}{\sqrt{V}} \sum_{\vec{k}} \nu_{\alpha}(\vec{k}) e^{i \vec{k} \cdot \vec{x}}, \\
& \nu_{\alpha}(\vec{k})=\sum_{\lambda}\left(\alpha_{\vec{k}, \lambda}^{(\alpha)} U_{\vec{k}, \lambda}^{(\alpha)}+\beta_{-\vec{k}, \lambda}^{(\alpha) \dagger} V_{-\vec{k}, \lambda}^{(\alpha)}\right)
\end{aligned}
$$

where the index $\lambda$ refers to the Dirac spin index and we have kept the same notation for the field and its spatial Fourier transform to avoid cluttering of notation. A flavor Fock representation is defined by choosing the spinors $U$ and $V$ respectively. In principle these spinors can be chosen to be the positive and negative energy solutions of a Dirac equation with an arbitrary mass, in what follows we will choose these to be $m_{e} ; m_{\mu}$, namely the masses of the flavor eigenstates in the absence of mixing. While we consider this to be a physically motivated choice, it is by no means unique and different alternatives have been discussed in the literature 40, 41, 42].

Thus the spinors $U$ and $V$ are chosen to be solutions of the following Dirac equations

$$
\begin{aligned}
& \gamma^{0}\left(\vec{\gamma} \cdot \vec{k}+m_{\alpha}\right) U_{\vec{k}, \lambda}^{(\alpha)}=\omega_{\alpha}(k) U_{\vec{k}, \lambda}^{(\alpha)} \\
& \gamma^{0}\left(\vec{\gamma} \cdot \vec{k}+m_{\alpha}\right) V_{-\vec{k}, \lambda}^{(\alpha)}=-\omega_{\alpha}(k) V_{-\vec{k}, \lambda}^{(\alpha)} \\
& \omega_{\alpha}(k)=\sqrt{k^{2}+m_{\alpha}^{2}}
\end{aligned}
$$

The Dirac spinors $U$ and $V$, are normalized as follows (no sum over the index $\alpha$ )

$$
U_{\vec{k}, \lambda}^{(\alpha) \dagger} U_{\vec{k}, \lambda^{\prime}}^{(\alpha)}=V_{\vec{k}, \lambda}^{(\alpha) \dagger} V_{\vec{k}, \lambda^{\prime}}^{(\alpha)}=\delta_{\lambda, \lambda^{\prime}} ; U_{\vec{k}, \lambda}^{(\alpha) \dagger} V_{-\vec{k}, \lambda^{\prime}}^{(\alpha)}=0
$$

and the creation and annihilation operators $\alpha_{\vec{k}, \lambda} ; \beta_{\vec{k}, \lambda}$ obey the usual canonical anticommutation relations.

On the other hand, upon quantization the field operators $\psi_{i}(x)$ associated with mass eigenstates at time $t=0$ are given by 


$$
\begin{aligned}
& \psi_{i}(\vec{x})=\frac{1}{\sqrt{V}} \sum_{k} \psi_{i}(\vec{k}) e^{i \vec{k} \cdot \vec{x}} \\
& \psi_{i}(\vec{k})=\sum_{\lambda}\left(a_{\vec{k}, \lambda}^{(i)} F_{\vec{k}, \lambda}^{(i)}+b_{-\vec{k}, \lambda}^{(i) \dagger} G_{-\vec{k}, \lambda}^{(i)}\right) .
\end{aligned}
$$

where the spinors $F, G$ are now solutions of the following Dirac equations

$$
\begin{aligned}
& \gamma^{0}\left(\vec{\gamma} \cdot \vec{k}+M_{i}\right) F_{\vec{k}, \lambda}^{(i)}=E_{i}(k) F_{\vec{k}, \lambda}^{(i)} \\
& \gamma^{0}\left(\vec{\gamma} \cdot \vec{k}+M_{i}\right) G_{-\vec{k}, \lambda}^{(i)}=-E_{i}(k) G_{-\vec{k}, \lambda}^{(i)} \\
& E_{i}(k)=\sqrt{k^{2}+M_{i}^{2}}
\end{aligned}
$$

with the normalization conditions (no sum over the label $i$ )

$$
F_{\vec{k}, \lambda}^{(i) \dagger} F_{\vec{k}, \lambda^{\prime}}^{(i)}=G_{\vec{k}, \lambda}^{(i) \dagger} G_{\vec{k}, \lambda^{\prime}}^{(i)}=\delta_{\lambda, \lambda^{\prime}} ; F_{\vec{k}, \lambda}^{(i) \dagger} G_{-\vec{k}, \lambda^{\prime}}^{(i)}=0 .
$$

Similarly, the operators $a$ and $b$ satisfy usual canonical anticommutation relations.

\section{A. Hamiltonian and Charges}

The total free field Hamiltonian for mixed neutrinos in the diagonal (mass) basis is given by

$$
H=\sum_{\vec{k}, i}\left[\bar{\psi}_{i}(\vec{k})\left(\vec{\gamma} \cdot \vec{k}+M_{i}\right) \psi_{i}(\vec{k})\right]=\sum_{\vec{k}, \lambda, i}\left(a_{\vec{k}, \lambda}^{(i) \dagger} a_{\vec{k}, \lambda}^{(i)}+b_{\vec{k}, \lambda}^{(i) \dagger} b_{\vec{k}, \lambda}^{(i)}-1\right) E_{i}(k),
$$

Therefore the time evolution of the operators $a, b$ is given by

$$
\begin{aligned}
a_{\vec{k}, \lambda}^{(i)}(t) & =a_{\vec{k}, \lambda}^{(i)} e^{-i E_{i}(k) t} \\
b_{\vec{k}, \lambda}^{(i)}(t) & =b_{\vec{k}, \lambda}^{(i)} e^{-i E_{i}(k) t} .
\end{aligned}
$$

The free field Lagrangian density (II.1) is invariant under independent phase transformations of the fields $\psi_{1,2}$, hence the individual $U(1)$ charges

$$
Q_{i}=\int d^{3} x \psi_{i}^{\dagger}(\vec{x}, t) \psi_{i}(\vec{x}, t)=\sum_{\vec{k}, \lambda}\left[a_{\vec{k}, \lambda}^{(i) \dagger} a_{\vec{k}, \lambda}^{(i)}-b_{\vec{k}, \lambda}^{(i) \dagger} b_{\vec{k}, \lambda}^{(i)}+1\right]
$$

are time independent.

The discussion that follows will focus on describing a statistical density matrix which is diagonal in the flavor basis and describes a hot and or dense ensemble of flavor neutrinos. This discussion requires the flavor Hamiltonian which is obtained from the Lagrangian density (III.1) for vanishing mixing $m_{e \mu}=0$, namely

$$
H_{f}=H_{e}+H_{\mu}=\sum_{\vec{k}, \alpha}\left[\bar{\nu}_{\alpha}(\vec{k})\left(\vec{\gamma} \cdot \vec{k}+m_{\alpha}\right) \nu_{\alpha}(\vec{k})\right]=\sum_{\vec{k}, \lambda, \alpha}\left(\alpha_{\vec{k}, \lambda}^{(\alpha) \dagger} \alpha_{\vec{k}, \lambda}^{(\alpha)}+\beta_{\vec{k}, \lambda}^{(\alpha) \dagger} \beta_{\vec{k}, \lambda}^{(\alpha)}-1\right) \omega_{\alpha}(k),
$$

The flavor Hamiltonian above is invariant under independent phase transformations of the flavor fields $\nu_{\alpha}$, thus the individual flavor charges commute with $H_{f}$

$$
q_{\alpha}=\int d^{3} x \nu_{\alpha}^{\dagger}(\vec{x}) \nu_{\alpha}(\vec{x})=\sum_{\vec{k}} \nu_{\alpha}^{\dagger}(\vec{k}) \nu_{\alpha}(\vec{k})=\sum_{\vec{k}, \lambda}\left[\alpha_{\vec{k}, \lambda}^{(\alpha) \dagger} \alpha_{\vec{k}, \lambda}^{(\alpha)}-\beta_{\vec{k}, \lambda}^{(\alpha) \dagger} \beta_{\vec{k}, \lambda}^{(\alpha)}+1\right]
$$


Using the transformation law (II.2) between flavor and mass eigenstates it is straightforward to find that the total charges are the same, namely

$$
\sum_{i, \vec{k}} \psi_{i}^{\dagger}(\vec{k}, t) \psi_{i}(\vec{k}, t)=\sum_{\alpha, \vec{k}} \nu_{\alpha}^{\dagger}(\vec{k}, t) \nu_{\alpha}(\vec{k}, t) \Rightarrow Q_{1}+Q_{2}=q_{e}+q_{\mu}
$$

\section{B. Density matrix and time evolution}

As stated in the introduction, our focus and goal is to study the time evolution of the distribution function of flavor neutrinos, at the level of free field theory at this stage. The question that we posed in the introduction and address here is the following: consider that at some given time the gas of flavor neutrinos and antineutrinos are described by a quantum statistical ensemble with a Fermi-Dirac distribution function with a fixed chemical potential for each flavor, namely

$$
n^{(\alpha)}(k)=\frac{1}{e^{\beta\left(\omega_{\alpha}(k)-\mu_{\alpha}\right)}+1} ; \bar{n}^{(\alpha)}(k)=\frac{1}{e^{\beta\left(\omega_{\alpha}(k)+\mu_{\alpha}\right)}+1}
$$

with $\beta=1 / T$ and $\mu_{\alpha}$ the chemical potential for each flavor.

Such an ensemble is described by a quantum statistical density matrix which is diagonal in the Fock space of flavor eigenstates and is given by

$$
\hat{\rho}=\hat{\rho}^{(e)} \otimes \hat{\rho}^{(\mu)}
$$

with the flavor density matrices

$$
\hat{\rho}^{(\alpha)}=e^{-\beta\left(H_{\alpha}-\mu_{\alpha} q_{\alpha}\right)}
$$

Hence the initial distribution functions are given by

$$
\begin{aligned}
\left\langle\alpha_{\vec{k}, \lambda}^{(\alpha) \dagger} \alpha_{\vec{k}, \lambda}^{(\alpha)}\right\rangle & =\operatorname{Tr} \hat{\rho}^{(\alpha)} \alpha_{\vec{k}, \lambda}^{(\alpha) \dagger} \alpha_{\vec{k}, \lambda}^{(\alpha)}=n^{(\alpha)}(k) \\
\left\langle\beta_{\vec{k}, \lambda}^{(\alpha) \dagger} \beta_{\vec{k}, \lambda}^{(\alpha)}\right\rangle & =\operatorname{Tr} \hat{\rho}^{(\alpha)} \beta_{\vec{k}, \lambda}^{(\alpha) \dagger} \beta_{\vec{k}, \lambda}^{(\alpha)}=\bar{n}^{(\alpha)}(k)
\end{aligned}
$$

In the expressions above we have assumed that the distribution of flavor neutrinos are spin independent, of course a spin dependence of the distribution function can be incorporated in the description.

Although we have stated the problem in terms of a gas flavor neutrinos in thermal equilibrium with Fermi-Dirac distributions, this restriction can be relaxed to arbitrary non-equilibrium single particle distributions consistent with Fermi-Dirac statistics. Regardless of the initial distributions the ensuing time evolution with the full Hamiltonian with mixing will be out of equilibrium.

\section{Cold degenerate case:}

The case of a cold, degenerate gas of neutrinos is described by the zero temperature limit but fixed chemical potential of the density matrix (II.21) with (II.22). In this limit the individual flavor neutrino gases form Fermi seas "filled up"

to the Fermi momentum $k_{F}^{(\alpha)}$. Consider the case of a positive chemical potential corresponding to a degenerate gas of neutrinos without antineutrinos at zero temperature, the degenerate ground state is given by

$$
|F S>=| F S>^{(e)} \otimes \mid F S>^{(\mu)}
$$

with

$$
\left|F S>^{(\alpha)}=\prod_{\vec{k}}^{k_{F}^{(\alpha)}} \alpha_{\vec{k}, \uparrow}^{(\alpha) \dagger} \alpha_{\vec{k}, \downarrow}^{(\alpha) \dagger}\right| 0>^{(\alpha)}
$$


with the flavor vacuum state $\mid 0>^{(\alpha)}$ annihilated by the destruction operators $\alpha_{\vec{k}, \lambda}^{(\alpha)} ; \beta_{\vec{k}, \lambda}^{(\alpha)}$. The initial density matrix in this case is that of a pure state

$$
\hat{\rho}=|F S><F S|
$$

the distribution function of flavor neutrinos is given by

$$
n^{(\alpha)}(k)=\Theta\left(k_{F}^{(\alpha)}-k\right), \bar{n}^{(\alpha)}(k)=0
$$

and the chemical potential is $\mu_{\alpha}=\omega_{\alpha}\left(k_{F}\right)$. The Fermi momentum is as usual given by

$$
k_{F}^{(\alpha)}=\left(3 \pi^{2} \mathcal{N}^{(\alpha)}\right)^{1 / 3} \Rightarrow k_{F}^{(\alpha)}(e V)=6.19\left(\frac{\mathcal{N}^{(\alpha)}}{10^{15} \mathrm{~cm}^{-3}}\right)^{1 / 3}
$$

with $\mathcal{N}^{(\alpha)}$ the neutrino density for each flavor. Although the zero temperature limit is described by a pure state, this state is a truly many body state

An important many body aspect of the situation under consideration can be gleaned by studying how the creation and annihilation operators of mass eigenstates act on the state $\mid F S>$. Consider for example the action of the annihilation operator $a_{\vec{k}, \lambda}^{(1)}$ on the state, to understand this question we must first obtain $a_{\vec{k}, \lambda}^{(1)}$ in terms of the creation and annihilation operators of flavor eigenstates. From equation (II.10) and the relation between fields given by (II.2) we find

$$
a_{\vec{k}, \lambda}^{(1)}=F_{\vec{k}, \lambda}^{(1), \dagger}\left[C \nu_{e}(\vec{k})-S \nu_{\mu}(\vec{k})\right]
$$

and the expansion for the flavor fields given by (II.7) clearly indicates that if $k<k_{F}^{\mu}<k_{F}^{e}$, for example, then $a_{\vec{k}, \lambda}^{(1)} \mid F S>$ is a superposition of states with an electron neutrino "hole", an electron antineutrino, a muon neutrino "hole" and a muon antineutrino. The antiparticle components of the wave function $a_{\vec{k}, \lambda}^{(1)} \mid F S>$ is a result of the non-vanishing overlap between the positive energy spinors for mass eigenstates and the negative energy spinors for flavor eigenstates [0].

\section{Time evolution}

Within the framework of free field theory of mixed neutrinos, the time evolution is completely determined by the total Hamiltonian $H$ given by eqn. (II.14).

In the Schroedinger picture the density matrix evolves in time with the full Hamiltonian as follows

$$
\hat{\rho}(t)=e^{-i H t} \hat{\rho}(0) e^{i H t}
$$

Since the full Hamiltonian $H$ does not commute with $H_{e}, H_{\mu}$ because of the flavor mixing, the density matrix does not commute with the Hamiltonian and therefore evolves in time. This is the statement that the initial density matrix (II.21) describes an ensemble out of equilibrium when flavor neutrinos are mixed.

Our goal is to obtain the time evolution of the distribution functions for flavor neutrinos and antineutrinos, namely

$$
n^{(\alpha)}(\vec{k}, t)=\operatorname{Tr} \hat{\rho}^{(\alpha)}(t) \alpha_{\vec{k}, \lambda}^{(\alpha) \dagger} \alpha_{\vec{k}, \lambda}^{(\alpha)}=\operatorname{Tr} \hat{\rho}^{(\alpha)}(0) \alpha_{\vec{k}, \lambda}^{(\alpha) \dagger}(t) \alpha_{\vec{k}, \lambda}^{(\alpha)}(t)
$$

and similarly for the antineutrino distribution function. The initial distribution functions $n^{(\alpha)}(\vec{k}, 0)=n^{(\alpha)}(\vec{k})($ and similarly for antineutrinos) given by equations (11.23) or (II.20) for the case of an initial thermal distribution.

It is more convenient to describe the time evolution in the Heisenberg picture wherein the density matrix does not depend on time and the Heisenberg field operators carry the time dependence as made explicit in eqn. (II.36). 
The free fields associated with the mass eigenstates $\psi_{i}$ evolve in time with the usual time dependent phases multiplying the creation and annihilation operators, namely

$$
\psi_{i}(\vec{k}, t)=e^{i H t} \psi_{i}(\vec{k}, 0) e^{-i H t}=\sum_{\lambda}\left(a_{\vec{k}, \lambda}^{(i)} e^{-i E_{i}(k) t} F_{\vec{k}, \lambda}^{(i)}+b_{-\vec{k}, \lambda}^{(i) \dagger} e^{i E_{i}(k) t} G_{-\vec{k}, \lambda}^{(i)}\right)
$$

The time evolution of the fields associated with flavor eigenstates, namely $\nu_{\alpha}$ is not so simple:

$$
\nu_{\alpha}(\vec{k}, t)=e^{i H t} \nu_{\alpha}(\vec{k}, 0) e^{-i H t}=\sum_{\lambda}\left(\alpha_{\vec{k}, \lambda}^{(\alpha)}(t) U_{\vec{k}, \lambda}^{(\alpha)}+\beta_{-\vec{k}, \lambda}^{(\alpha) \dagger}(t) V_{-\vec{k}, \lambda}^{(\alpha)}\right)
$$

where the time dependent operators $\alpha_{\vec{k}, \lambda}^{(\alpha)}(t) ; \beta_{-\vec{k}, \lambda}^{(\alpha) \dagger}(t)$ can be obtained by writing the flavor fields in terms of the mass eigenstate fields using eqn. (II.2) and projecting out the components using the orthogonality property given by eqn. (II.9), leading for example to

$$
\begin{aligned}
\alpha_{\vec{k}, \lambda}^{(e)}(t) & =U_{\vec{k}, \lambda}^{(e) \dagger}\left[C \psi_{1}(\vec{k}, t)+S \psi_{2}(\vec{k}, t)\right] \\
\beta_{-\vec{k}, \lambda}^{(e) \dagger}(t) & =V_{-\vec{k}, \lambda}^{(e) \dagger}\left[C \psi_{1}(\vec{k}, t)+S \psi_{2}(\vec{k}, t)\right]
\end{aligned}
$$

The expression (II.34) reveals several subtle aspects which are highlighted by considering in detail for example the time evolution of the operator that creates electron neutrinos (a similar analysis holds for the muon neutrinos and their respective antiparticles)

$$
\begin{aligned}
\alpha_{\vec{k}, \lambda}^{(e) \dagger}(t)= & \sum_{\lambda^{\prime}}\left\{\left(C a_{\vec{k}, \lambda^{\prime}}^{(1) \dagger} e^{i E_{1}(k) t} F_{\vec{k}, \lambda^{\prime}}^{(1) \dagger} U_{\vec{k}, \lambda}^{(e)}+S a_{\vec{k}, \lambda^{\prime}}^{(2) \dagger} e^{i E_{2}(k) t} F_{\vec{k}, \lambda^{\prime}}^{(2) \dagger} U_{\vec{k}, \lambda}^{(e)}\right)+\right. \\
& \left.\left(C b_{-\vec{k}, \lambda^{\prime}}^{(1)} e^{-i E_{1}(k) t} G_{-\vec{k}, \lambda^{\prime}}^{(1) \dagger} U_{\vec{k}, \lambda}^{(e)}+S b_{-\vec{k}, \lambda^{\prime}}^{(2)} e^{-i E_{2}(k) t} G_{-\vec{k}, \lambda^{\prime}}^{(2) \dagger} U_{\vec{k}, \lambda}^{(e)}\right)\right\}
\end{aligned}
$$

It is a simple and straightforward exercise using the completeness and orthogonality of the respective spinor wavefunctions, to show that the creation and annihilation operators of flavor states indeed fulfill the canonical anticommutation relations. A Fock representation of flavor states is therefore consistent and moreover needed to describe a quantum statistical ensemble of flavor neutrinos.

The first line in the above expression shows that the annihilation operator for electron corresponds to the expected combination of creation operators for mass eigenstates multiplied by the cosine and sine of the mixing angle, but also multiplied by the overlap of the different spinor wavefunctions. Furthermore, the electron creation operator also involves the annihilation of antiparticles associated with the mass eigenstates, a feature recognized in ref. [40]. There are two important consequences of the exact relation (II.35):

- The amplitude for creating a mass eigenstate out of the vacuum of mass eigenstates by an electron neutrino creation operator is not only given by the cosine or sine (respectively) of the mixing angle, but also by the overlap of the spinor wave functions $F_{\vec{k}, \lambda^{\prime}}^{(i) \dagger} U_{\vec{k}, \lambda}^{(e)}$.

- The electron neutrino creation operator destroys antiparticle mass eigenstates. While this aspect is not relevant when the electron neutrino creation operator acts on the vacuum of mass eigenstates, it becomes relevant in a medium where both particles and antiparticles states are populated.

These aspects, which were also highlighted in references 40, 41, 42] will be at the heart of the subtle many body aspects of neutrino mixing which contribute to the time evolution of the distribution functions studied below.

The time dependent distribution functions are obtained by taking the trace with the initial density matrix

$$
n^{(\alpha)}(\vec{k}, t)=\operatorname{Tr} \hat{\rho}^{(\alpha)}(0) \alpha_{\vec{k}, \lambda}^{(\alpha) \dagger}(t) \alpha_{\vec{k}, \lambda}^{(\alpha)}(t)
$$

and similarly for the other distribution functions. One can use the expression (II.35) for the time evolution of the Heisenberg field operator (and the equivalent for the hermitian conjugate), however in order to compute the time evolved distribution function we would need to compute the expectation value of bilinears of the field operators $\psi_{i}$ in 
the flavor diagonal density matrix $\hat{\rho}(0)$. To do this we would have to re-write the creation and annihilation operators $a_{\vec{k}, \lambda}^{(i)} ; b_{\vec{k}, \lambda}^{(i)} ;$ etc. in the expression (11.35) back in terms of the creation and annihilation operators $\alpha_{\vec{k}, \lambda}^{(\alpha)} ; \beta_{\vec{k}, \lambda}^{(\alpha)} ;$ etc.. This is obviously a rather cumbersome method. A more systematic manner to carry out this program is presented below.

Using the expressions (11.17II.18) we find the following identities

$$
\begin{aligned}
& \frac{1}{2}\left\langle\bar{\nu}_{\alpha}(\vec{k}, t) \gamma^{0} \nu_{\alpha}(\vec{k}, t)\right\rangle=n^{(\alpha)}(\vec{k}, t)-\bar{n}^{(\alpha)}(\vec{k}, t)+1 \\
& \frac{1}{2 \omega_{\alpha}(k)}\left\langle\bar{\nu}_{\alpha}(\vec{k}, t)\left(\vec{\gamma} \cdot \vec{k}+m_{\alpha}\right) \nu_{\alpha}(\vec{k}, t)\right\rangle=n^{(\alpha)}(\vec{k}, t)+\bar{n}^{(\alpha)}(\vec{k}, t)-1
\end{aligned}
$$

Thus the computation of the distribution functions or combinations of them requires to find general expressions of the form

$$
<\bar{\nu}_{e}(\vec{k}, t) \mathcal{O} \nu_{e}(\vec{k}, t)>=\mathcal{O}_{f g}<\left[\bar{\nu}_{e}(\vec{k}, t)\right]_{f}\left[\nu_{e}(\vec{k}, t)\right]_{g}>.
$$

where the Dirac indices $f, g$ are summed over and the averages are in the flavor diagonal density matrix (II.21III.22).

Since the time evolution of the fields $\psi_{i}$ is that of usual free Dirac field in terms of positive and negative frequency components, we write

$$
\psi_{(i)}(\vec{k}, t)=\left(\Lambda_{+}^{(i)}(\vec{k}) e^{-i E_{i} t}+\Lambda_{-}^{(i)}(\vec{k}) e^{i E_{i} t}\right) \psi_{(i)}(\vec{k}, 0) .
$$

Where we have introduced the positive and negative frequency projector operators $\Lambda_{+}(k)$ and $\Lambda_{-}(k)$ respectively which are given by

$$
\begin{aligned}
\Lambda_{+}^{(i)}(\vec{k}) & =\sum_{\lambda} F_{\vec{k}, \lambda}^{(i)} F_{\vec{k}, \lambda}^{(i) \dagger}=\left(\frac{\not k_{(i)}+M_{i}}{2 E_{i}}\right) \gamma^{0}, \\
\Lambda_{-}^{(i)}(\vec{k}) & =\sum_{\lambda} G_{-\vec{k}, \lambda}^{(i)} G_{-\vec{k}, \lambda}^{(i) \dagger}=\gamma^{0}\left(\frac{\not k_{(i)}-M_{i}}{2 E_{i}}\right) \\
\not k_{(i)} & =\gamma^{0} E_{i}(k)-\vec{\gamma} \cdot \vec{k}
\end{aligned}
$$

These projection operators have the following properties,

$$
\begin{aligned}
& \Lambda_{+}^{(i) \dagger}(\vec{k})=\Lambda_{+}^{(i)}(\vec{k}) ; \quad \Lambda_{-}^{(i) \dagger}(\vec{k})=\Lambda_{-}^{(i)}(\vec{k}) \\
& \Lambda_{+}^{(i)}(\vec{k}) \Lambda_{-}^{(i)}(\vec{k})=0 ; \quad \Lambda_{-}^{(i)}(\vec{k}) \Lambda_{+}^{(i)}(\vec{k})=0 \\
& \Lambda_{+}^{(i)}(\vec{k})+\Lambda_{-}^{(i)}(\vec{k})=1 .
\end{aligned}
$$

We can now write the time evolution of the flavor fields in a rather simple manner by using the relations between the fields given by (II.2) and the inverse relation which allows to write $\psi_{i}(\vec{k}, 0)$ in (II.40) back in terms of $\nu_{\alpha}(\vec{k}, 0)$. We find

$$
\begin{aligned}
& \psi_{1}(\vec{k}, t)=\gamma^{0} F_{1}(\vec{k}, t)\left[C \nu_{e}(\vec{k}, 0)-S \nu_{\mu}(\vec{k}, 0)\right] \\
& \bar{\psi}_{1}(\vec{k}, t)=\left[C \bar{\nu}_{e}(\vec{k}, 0)-S \bar{\nu}_{\mu}(\vec{k}, 0)\right] \tilde{F}_{1}(\vec{k}, t) \gamma^{0} \\
& \psi_{2}(\vec{k}, t)=\gamma^{0} F_{2}(\vec{k}, t)\left[C \nu_{\mu}(\vec{k}, 0)+S \nu_{e}(\vec{k}, 0)\right] \\
& \bar{\psi}_{2}(\vec{k}, t)=\left[C \bar{\nu}_{\mu}(\vec{k}, 0)+S \bar{\nu}_{e}(\vec{k}, 0)\right] \tilde{F}_{2}(\vec{k}, t) \gamma^{0}
\end{aligned}
$$

Where we have introduced the following time evolution kernels

$$
\begin{aligned}
F_{j}(\vec{k}, t) & =\gamma^{0}\left[\Lambda_{+}^{(j)}(\vec{k}) e^{-i E_{j}(k) t}+\Lambda_{-}^{(j)}(\vec{k}) e^{i E_{j}(k) t}\right], \\
\tilde{F}_{j}(\vec{k}, t) & =F_{j}(\vec{k},-t) \gamma^{0} ; j=1,2 .
\end{aligned}
$$


After straightforward algebra using the mixing transformation (II.2) and equations (II.47 II.50) we find the following result for the time evolution of the flavor fields

$$
\begin{aligned}
\nu_{e}(k, t) & =T_{e e}(\vec{k}, t) \nu_{e}(\vec{k}, 0)+T_{e \mu}(\vec{k}, t) \nu_{\mu}(\vec{k}, 0), \\
\bar{\nu}_{e}(k, t) & =\bar{\nu}_{e}(\vec{k}, 0) \tilde{T}_{e e}(\vec{k}, t)+\bar{\nu}_{\mu}(\vec{k}, 0) \tilde{T}_{e \mu}(\vec{k}, t), \\
\nu_{\mu}(\vec{k}, t) & =T_{\mu \mu}(\vec{k}, t) \nu_{\mu}(\vec{k}, 0)+T_{\mu e}(\vec{k}, t) \nu_{e}(\vec{k}, 0), \\
\bar{\nu}_{\mu}(\vec{k}, t) & =\bar{\nu}_{\mu}(\vec{k}, 0) \tilde{T}_{\mu \mu}(\vec{k}, t)+\bar{\nu}_{e}(\vec{k}, 0) \tilde{T}_{\mu e}(\vec{k}, t),
\end{aligned}
$$

where the time evolution operators are given by

$$
\begin{aligned}
T_{e e}(\vec{k}, t) & =\gamma^{0}\left[C^{2} F_{1}(\vec{k}, t)+S^{2} F_{2}(\vec{k}, t)\right], \\
T_{\mu \mu}(\vec{k}, t) & =\gamma^{0}\left[C^{2} F_{2}(\vec{k}, t)+S^{2} F_{1}(\vec{k}, t)\right], \\
T_{e \mu}(\vec{k}, t) & =T_{\mu e}=C S \gamma^{0}\left[F_{2}(\vec{k}, t)-F_{1}(\vec{k}, t)\right] \\
\tilde{T}_{\alpha \beta}(\vec{k}, t) & =\gamma^{0} T_{\alpha \beta}(\vec{k},-t) \gamma^{0},
\end{aligned}
$$

Furthermore since the initial density matrix is flavor diagonal, we find the following expectation values

$$
\begin{aligned}
<\left[\bar{\nu}_{e}(\vec{k}, t)\right]_{f}\left[\nu_{e}(\vec{k}, t)\right]_{g}>= & <\left[\bar{\nu}_{e}(\vec{k}, 0)\right]_{r}\left[\nu_{e}(\vec{k}, 0)\right]_{s}>\left[\tilde{T}_{e e}(\vec{k}, t)\right]_{r f}\left[T_{e e}(\vec{k}, t)\right]_{g s} \\
& +<\left[\bar{\nu}_{\mu}(\vec{k}, 0)\right]_{r}\left[\nu_{\mu}(\vec{k}, 0)\right]_{s}>\left[\tilde{T}_{e \mu}(\vec{k}, t)\right]_{r f}\left[T_{e \mu}(\vec{k}, t)\right]_{g s},
\end{aligned}
$$

and similarly for the muon neutrino fields, where $\langle\cdots\rangle$ stands for the trace with the initial density matrix.

A noteworthy feature of the above exact expressions is that the time evolution of the flavor neutrino fields mix positive and negative frequency components of the mass eigenstates. Namely a flavor neutrino state is a linear combination of particles and antiparticles of mass eigenstates. Thus a wave packet of flavor neutrinos will necessarily mix positive and negative frequencies of mass eigenstates. This mixing between particles and antiparticles is a consequence of the fact that a flavor eigenstate is a squeezed state of mass eigenstates and viceversa [40].

A simple calculation yields the following expectation values in the initial density matrix

$$
\begin{aligned}
<\left[\bar{\nu}_{\alpha}(\vec{k}, 0)\right]_{r}\left[\nu_{\alpha}(\vec{k}, 0)\right]_{s}> & =\left[\sum_{\lambda}<\alpha_{\vec{k}, \lambda}^{(\alpha) \dagger} \alpha_{\vec{k}, \lambda}^{(\alpha)}>\left[\bar{U}_{\vec{k}, \lambda}^{(\alpha)}\right]_{r}\left[U_{\vec{k}, \lambda}^{(\alpha)}\right]_{s}+\sum_{\lambda}<\beta_{-\vec{k}, \lambda}^{(\alpha) \dagger} \beta_{-\vec{k}, \lambda}^{(\alpha)}>\left[\bar{V}_{-\vec{k}, \lambda}^{(\alpha)}\right]_{r}\left[V_{-\vec{k}, \lambda}^{(\alpha)}\right]_{s}\right] \\
& =n^{(\alpha)}(k)\left(\frac{\not k_{\alpha}+m_{\alpha}}{2 \omega_{\alpha}(k)}\right)_{s r}+\left(1-\bar{n}^{(\alpha)}(k)\right)\left[\gamma^{0} \frac{\not k_{\alpha}-m_{\alpha}}{2 \omega_{\alpha}(k)} \gamma^{0}\right]_{s r} \equiv\left[N_{\alpha}(\vec{k})\right]_{s r} \\
k_{\alpha} & =\gamma^{0} \omega^{\alpha}(k)-\vec{\gamma} \cdot \vec{k}
\end{aligned}
$$

where $n^{\alpha}(k) ; \bar{n}^{\alpha}(k)$ are given by the expressions (II.20) and there are no flavor off-diagonal matrix elements at $t=0$ because the initial density matrix is flavor diagonal.

Combining all the above results, we find the final compact form for the time dependent expectation values in eqn. (II.39), namely

$$
<\bar{\nu}_{e}(\vec{k}, t) \mathcal{O} \nu_{e}(\vec{k}, t)>=\operatorname{Tr}\left[N_{e}(\vec{k}) \tilde{T}_{e e}(\vec{k}, t) \mathcal{O} T_{e e}(\vec{k}, t)\right]+\operatorname{Tr}\left[N_{\mu}(\vec{k}) \tilde{T}_{e \mu}(\vec{k}, t) \mathcal{O} T_{e \mu}(\vec{k}, t)\right]
$$

\section{Exact time evolution of distribution functions}

The exact time evolution (in free field theory) of flavor neutrinos is given by

$$
n^{(e)}(k, t) \equiv I^{(e)}(k, t)+J^{(e)}(k, t)
$$


where $I^{(e)}(k, t)$ and $J^{(e)}(k, t)$ are given by

$$
\begin{gathered}
I^{(e)}(k, t)=\frac{1}{4 \omega_{e}(k)} \operatorname{Tr}\left[N_{e}(k) \tilde{T}_{e e}(\vec{k}, t) \gamma^{0}\left(\not k_{e}+m_{e}\right) \gamma^{0} T_{e e}(\vec{k}, t)\right] \\
J^{(e)}(k, t)=\frac{1}{4 \omega_{e}(k)} \operatorname{Tr}\left[N_{\mu}(\vec{k}) \tilde{T}_{e \mu}(\vec{k}, t) \gamma^{0}\left(\not k_{e}+m_{e}\right) \gamma^{0} T_{e \mu}(\vec{k}, t)\right] . \\
\bar{n}^{(e)}(k, t)=1-\bar{I}^{(e)}(k, t)-\bar{J}^{(e)}(k, t)
\end{gathered}
$$

where $\bar{I}^{(e)}(k, t)$ and $\bar{J}^{(e)}(k, t)$ are given by

$$
\begin{aligned}
& \bar{I}^{(e)}(k, t)=\frac{1}{4 \omega_{e}(k)} \operatorname{Tr}\left[N_{e}(\vec{k}) \tilde{T}_{e e}(\vec{k}, t)\left(\not k_{e}-m_{e}\right) T_{e e}(\vec{k}, t)\right], \\
& \bar{J}^{(e)}(k, t)=\frac{1}{4 \omega_{e}(k)} \operatorname{Tr}\left[N_{\mu}(\vec{k}) \tilde{T}_{e \mu}(\vec{k}, t)\left(\not k_{e}-m_{e}\right) T_{e \mu}(\vec{k}, t)\right] .
\end{aligned}
$$

For the muon neutrinos and antineutrinos

$$
n^{(\mu)}(k, t)=I^{(\mu)}(k, t)+J^{(\mu)}(k, t)
$$

where $I^{(\mu)}(k, t)$ and $J^{(\mu)}(k, t)$ are given by

$$
\begin{gathered}
I^{(\mu)}(k, t)=\frac{1}{4 \omega_{\mu}(k)} \operatorname{Tr}\left[N_{\mu}(\vec{k}) \tilde{T}_{\mu \mu}(\vec{k}, t) \gamma^{0}\left(\not k_{\mu}+m_{\mu}\right) \gamma^{0} T_{\mu \mu}(\vec{k}, t)\right], \\
J^{(\mu)}(k, t)=\frac{1}{4 \omega_{\mu}(k)} \operatorname{Tr}\left[N_{e}(\vec{k}) \tilde{T}_{\mu e}(\vec{k}, t) \gamma^{0}\left(\not k_{\mu}+m_{\mu}\right) \gamma^{0} T_{\mu e}(\vec{k}, t)\right] . \\
\bar{n}^{(\mu)}(k, t)=1-\bar{I}^{(\mu)}(k, t)-\bar{J}^{(\mu)}(k, t),
\end{gathered}
$$

where $\bar{I}^{(\mu)}(k, t)$ and $\bar{J}^{(\mu)}(k, t)$ are given by

$$
\begin{aligned}
& \bar{I}^{(\mu)}(k, t)=\frac{1}{4 \omega_{\mu}(k)} \operatorname{Tr}\left[N_{\mu}(\vec{k}) \tilde{T}_{\mu \mu}(\vec{k}, t)\left(\not k_{\mu}-m_{\mu}\right) T_{\mu \mu}(\vec{k}, t)\right], \\
& \bar{J}^{(\mu)}(k, t)=\frac{1}{4 \omega_{\mu}(k)} \operatorname{Tr}\left[N_{e}(\vec{k}) \tilde{T}_{\mu e}(\vec{k}, t)\left(\not k_{\mu}-m_{\mu}\right) T_{\mu e}(\vec{k}, t)\right] .
\end{aligned}
$$

The calculation of the traces is simplified by the observation that all of the different terms that enter in the trace, such as $N_{\alpha}(\vec{k}) ; \tilde{T}_{\alpha, \alpha^{\prime}}(\vec{k}, t) \gamma^{0} ; \gamma^{0} T_{\alpha, \alpha^{\prime}}(\vec{k}, t)$ can be written in the form

$$
\gamma^{0} A_{0}(\vec{k}, t)-\vec{\gamma} \cdot \vec{A}(\vec{k}, t)+B(\vec{k}, t) \equiv \not A(\vec{k}, t)+B(\vec{k}, t)
$$

where the coefficient functions $A_{0}(\vec{k}, t) ; \vec{A}(\vec{k}, t) ; B(\vec{k}, t)$ can be read off each individual term. Thus the traces in the terms above can be calculated by using the standard formulae for the traces of two and four Dirac matrices.

\section{E. Fast and slow time scales}

While the exact compact expressions above describe the full time evolution and provide a set of closed form expressions, they hide the fact that there two widely different time scales. These different time scales can be revealed 
by unravelling the different contributions to the distribution functions as follows. Consider the expectation value on the right hand side of eqn. (11.39) for the case of the electron neutrino

$$
\begin{aligned}
<\left[\bar{\nu}_{e}(\vec{k}, t)\right]_{f}\left[\nu_{e}(\vec{k}, t)\right]_{g}> & =C^{2}<\left[\bar{\psi}_{1}(\vec{k}, t)\right]_{f}\left[\psi_{1}(\vec{k}, t)\right]_{g}>+S^{2}<\left[\bar{\psi}_{2}(\vec{k}, t)\right]_{f}\left[\psi_{2}(\vec{k}, t)\right]_{g}> \\
& +C S<\left[\bar{\psi}_{1}(\vec{k}, t)\right]_{f}\left[\psi_{2}(\vec{k}, t)\right]_{g}+\left[\bar{\psi}_{2}(\vec{k}, t)\right]_{f}\left[\psi_{1}(\vec{k}, t)\right]_{g}>
\end{aligned}
$$

the case of the muon neutrino can be obtained from the expression above by replacing $S \rightarrow C ; C \rightarrow-S$.

By writing each one of the fields $\psi_{i}$ in terms of the positive and negative frequency contributions which evolve in time with the phases $e^{\mp i E_{i}(k) t}$ respectively, it is clear that in the products $\bar{\psi}_{i}(\vec{k}, t) \psi_{i}(\vec{k}, t)$ there is a contribution that does not depend on time and terms that oscillate in time with the phases $e^{\mp 2 i E_{i}(k) t}$. These oscillatory terms arise from the interference between particles and antiparticles akin to zitterbewegung in principle do not vanish when the density matrix is diagonal in the flavor basis. In the general expectation values in eqn (II.39) these oscillatory terms will multiply the matrix elements of the form $\bar{F}_{\vec{k}, \lambda}^{(i)} \mathcal{O} G_{-\vec{k}, \lambda}^{(i)}$, thus if these matrix elements do not vanish, these oscillatory terms are present. In the second line in eqn. (II.79) a similar argument shows that there are two types of oscillatory terms, $e^{\mp i\left(E_{1}(k)+E_{2}(k)\right) t}$ and $e^{\mp i\left(E_{1}(k)-E_{2}(k)\right) t}$. The former arise from the interference between the particle and antiparticle states of different masses, while the latter from interference between particle states of different masses (or antiparticle).

The combined analysis from solar neutrinos and KamLAND 45] suggest that for two flavor mixing $M_{1}^{2}-M_{2}^{2}=$ $\Delta M^{2} \sim 7 \times 10^{-5}(\mathrm{eV})^{2}$ and cosmological constraints from WMAP [46] suggest that the average mass of neutrinos is $\bar{M} \lesssim 0.23 \mathrm{eV}$. Therefore even in the non-relativistic limit with $k \ll M_{i}$ the ratio $\left|E_{1}(k)-E_{2}(k)\right| /\left(E_{1}(k)+E_{2}(k)\right)<10^{-4}$ and certainly much smaller in the relativistic limit $k \gg M_{i}$. Hence because of the near degeneracy, or in the relativistic limit for any value of the masses, there are two widely different time scales of evolution for the flavor distribution functions. The longest one corresponding to the interference between particle states (or antiparticle states) of different masses while the shortest one corresponds to the interference between particle and antiparticle states of equal or different masses. This point will be revisited below.

The evolution of the flavor (lepton) asymmetry highlights these time scales clearly and is studied below.

\section{DEGENERATE GAS OF NEUTRINOS: EVOLUTION OF FLAVOR ASYMMETRY}

The results obtained above are general and valid for any temperature and chemical potential (density). In this section we focus on understanding the time evolution of the flavor asymmetry $n^{(\alpha)}(k, t)-\bar{n}^{(\alpha)}(k, t)$ in the case of a cold, degenerate gas of flavor neutrinos. From equations (11.37) and (1I.79) we find

$$
\begin{aligned}
n^{(e)}(\vec{k}, t)-\bar{n}^{(e)}(\vec{k}, t) & =\frac{C^{2}}{2}<\psi_{1}^{\dagger}(\vec{k}, t) \psi_{1}(\vec{k}, t)>+\frac{S^{2}}{2}<\psi_{2}^{\dagger}(\vec{k}, t) \psi_{2}(\vec{k}, t)>-1 \\
& +\frac{C S}{2}<\psi_{1}^{\dagger}(\vec{k}, t) \psi_{2}(\vec{k}, t)+\psi_{2}^{\dagger}(\vec{k}, t) \psi_{1}(\vec{k}, t)> \\
n^{(\mu)}(\vec{k}, t)-\bar{n}^{(\mu)}(\vec{k}, t) & =\frac{S^{2}}{2}<\psi_{1}^{\dagger}(\vec{k}, t) \psi_{1}(\vec{k}, t)>+\frac{C^{2}}{2}<\psi_{2}^{\dagger}(\vec{k}, t) \psi_{2}(\vec{k}, t)>-1 \\
& -\frac{C S}{2}<\psi_{1}^{\dagger}(\vec{k}, t) \psi_{2}(\vec{k}, t)+\psi_{2}^{\dagger}(\vec{k}, t) \psi_{1}(\vec{k}, t)>
\end{aligned}
$$

The first line of the expressions above is time independent because the overlap between positive and negative frequency components vanishes, and the time dependence arises solely from the interference between different mass eigenstates. The time dependent terms (second lines in the above expressions) are opposite for the two flavors realizing the fact that the total charge of mass eigenstates equals that of flavor eigenstates and is time independent (see eqn. (II.19)).

Furthermore the expectation values $<\psi_{i}^{\dagger}(\vec{k}, t) \psi_{i}(\vec{k}, t)>$ (no sum on $i$ ) are time independent (in the case of free field theory under consideration) since the interference term between positive and negative frequency spinors vanishes. The time dependence is completely encoded in the contribution that mixes the mass eigenstates.

Therefore the time dependence of the flavor asymmetry is completely determined by the quantity

$$
\chi(\vec{k}, t) \equiv \frac{C S}{2}<\left(\psi_{1}^{\dagger}(\vec{k}, t) \psi_{2}(\vec{k}, t)+\psi_{2}^{\dagger}(\vec{k}, t) \psi_{1}(\vec{k}, t)\right)>
$$


Using equations (II.47)- (II.50), it follows that

$$
\chi(\vec{k}, t)=\frac{C^{2} S^{2}}{2} \operatorname{Tr}\left[\left[N_{e}(\vec{k})-N_{\mu}(\vec{k})\right]\left[\tilde{F}_{1}(\vec{k}, t) F_{2}(\vec{k}, t)+\tilde{F}_{2}(\vec{k}, t) F_{1}(\vec{k}, t)\right]\right],
$$

The computation of the traces is simplified by writing

$$
\begin{aligned}
F_{j}(k, t) & =P_{j}(t)+M_{j}(t) \\
P_{j}^{0}(t) & =\cos \left(E_{j}(k) t\right), \\
\vec{P}_{j}(t) & =\frac{i \vec{k}}{E_{j}(k)} \sin \left(E_{j}(k) t\right), \\
M_{j}(t) & =-\frac{i M_{j}}{E_{j}(k)} \sin \left(E_{j}(k) t\right) .
\end{aligned}
$$

and similarly we write

$$
\begin{aligned}
N_{\alpha}(k) & =Q_{\alpha}+\widetilde{M}_{\alpha} \\
Q_{\alpha} & =\gamma^{0} Q_{\alpha}^{0}-\vec{\gamma} \cdot \vec{Q}_{\alpha}, \\
Q_{\alpha}^{0} & =\frac{1}{2}\left[n^{(\alpha)}(k)+1-\bar{n}^{(\alpha)}(k)\right], \\
\vec{Q}_{\alpha} & =\frac{\vec{k}}{2 \omega_{\alpha}(k)}\left[n^{(\alpha)}(k)-1+\bar{n}^{(\alpha)}(k)\right], \\
\widetilde{M}_{\alpha} & =\frac{m_{\alpha}}{2 \omega_{\alpha}(k)}\left[n^{(\alpha)}(k)-1+\bar{n}^{(\alpha)}(k)\right] .
\end{aligned}
$$

For further convenience, we define

$$
\Delta \emptyset=Q_{e}-\emptyset_{\mu}, \quad ; \quad \Delta \widetilde{M}=\widetilde{M}_{e}-\widetilde{M}_{\mu},
$$

in terms of which we obtain

$$
\begin{aligned}
\chi(\vec{k}, t)= & \frac{C^{2} S^{2}}{2} \operatorname{Tr}\left[(\Delta \varnothing+\Delta \widetilde{M})\left(P_{1}(-t)+M_{1}(-t)\right) \gamma^{0}\left(P_{2}(t)+M_{2}(t)\right)\right. \\
& \left.+(\Delta \varnothing+\Delta \widetilde{M})\left(P_{2}(-t)+M_{2}(-t)\right) \gamma^{0}\left(P_{1}(t)+M_{1}(t)\right)\right] .
\end{aligned}
$$

After some lengthy but straigthforward algebra we find

$$
\begin{aligned}
\chi(\vec{k}, t)= & \chi(\vec{k}, 0)-2 C^{2} S^{2}\left[\left(n^{(e)}(k)-\bar{n}^{(e)}(k)\right)-\left(n^{(\mu)}(k)-\bar{n}^{(\mu)}(k)\right)\right]\left[\left(1-\frac{k^{2}+M_{1} M_{2}}{E_{1}(k) E_{2}(k)}\right) \sin ^{2}\left(\frac{E_{1}(k)+E_{2}(k)}{2} t\right)+\right. \\
& \left.\left(1+\frac{k^{2}+M_{1} M_{2}}{E_{1}(k) E_{2}(k)}\right) \sin ^{2}\left(\frac{E_{1}(k)-E_{2}(k)}{2} t\right)\right],
\end{aligned}
$$

where $\chi(\vec{k}, 0)$ is given by

$$
\chi(\vec{k}, 0)=2 C^{2} S^{2}\left[\left(n^{(e)}(k)-\bar{n}^{(e)}(k)\right)-\left(n^{(\mu)}(k)-\bar{n}^{(\mu)}(k)\right)\right] .
$$

The expression (III.16) for the time dependence of the flavor asymmetry clearly shows that neutrino mixing results in a time evolution of the flavor asymmetry unless the flavor asymmetry for both flavors is the same. This is obviously 
a consequence of Pauli blocking: if the neutrino states are occupied up to the same momentum electron neutrinos cannot transform into an (occupied) muon neutrino state and viceversa.

In the case of a cold, degenerate gas of flavor neutrinos (we assume here both chemical potentials to be positive) is given by

$$
n^{(\alpha)}(k) \rightarrow \Theta\left(k_{F}^{(\alpha)}-k\right) ; \bar{n}^{(\alpha)}(k) \rightarrow 0
$$

If the chemical potential is different for the different flavors, the expression above shows that each wavevector mode will evolve with a different frequency and as a consequence of free field evolution there is no mode mixing. The important question is what is the time evolution of the total charge which is the integral of the flavor asymmetry over all momenta. This time evolution will be a result of the dephasing through the oscillations between different modes that are not Pauli blocked.

We now proceed to study analytically and numerically the time evolution of the flavor charge densities $q_{\alpha} / V$ with $q_{\alpha}$ given by eqn. (II.18) and $V$ the volume. We begin by defining

$$
\bar{M} \equiv \frac{M_{1}+M_{2}}{2} ; \Delta M^{2} \equiv M_{1}^{2}-M_{2}^{2}
$$

so that $M_{1}$ and $M_{2}$ can be written in terms of $\bar{M}$ and $\Delta M^{2}$ as

$$
M_{1}=\bar{M}\left(1+\frac{\Delta M^{2}}{4 \bar{M}^{2}}\right) \quad ; \quad M_{2}=\bar{M}\left(1-\frac{\Delta M^{2}}{4 \bar{M}^{2}}\right)
$$

We take the following as representative values for the two flavor case [9, 10] $\bar{M} \simeq 0.25 \mathrm{eV}$ and $\Delta M^{2} \simeq 7 \times 10^{-5}(\mathrm{eV})^{2}$. In what follows we assume that $k_{F}^{e}>k_{F}^{\mu}$ and introduce dimensionless variables by taking $k_{F}^{e}$ as the common scale, the opposite limit for the Fermi momenta can be obtained simply from the results below. Hence we define

$$
\begin{aligned}
q & =\frac{k}{k_{F}^{e}} ; q_{r}=\frac{k_{F}^{\mu}}{k_{F}^{e}} ; \tau=k_{F}^{e} t, \\
\bar{m} & =\frac{\bar{M}}{k_{F}^{e}} ; \delta m^{2}=m_{1}^{2}-m_{2}^{2}=\frac{M_{1}^{2}-M_{2}^{2}}{\left(k_{F}^{e}\right)^{2}} \\
m_{1} & =\bar{m}\left(1+\frac{\Delta M^{2}}{4 \bar{M}^{2}}\right) ; m_{2}=\bar{m}\left(1-\frac{\Delta M^{2}}{4 \bar{M}^{2}}\right) ; \\
\varepsilon_{1} & =\sqrt{q^{2}+m_{1}^{2}} ; \varepsilon_{2}=\sqrt{q^{2}+m_{2}^{2}} .
\end{aligned}
$$

Hence, in terms of $\mathcal{N}^{(\alpha)}=\left(k_{F}^{\alpha}\right)^{3} / 3 \pi^{2}$ (see eqn. (ㅍ.28), we find that the time evolution of the flavor charge densities are given by

$$
\begin{aligned}
& \frac{q_{e}(t)}{V}=\mathcal{N}^{(e)}-6 C^{2} S^{2} \mathcal{N}^{(e)}\left(I_{f}(\tau)+I_{s}(\tau)\right) \\
& \frac{q_{\mu}(t)}{V}=\mathcal{N}^{(\mu)}+6 C^{2} S^{2} \mathcal{N}^{(e)}\left(I_{f}(\tau)+I_{s}(\tau)\right)
\end{aligned}
$$

where

$$
\begin{aligned}
& I_{f}(\tau)=\int_{q_{r}}^{1} d q q^{2}\left(1-\frac{q^{2}+m_{1} m_{2}}{\varepsilon_{1} \varepsilon_{2}}\right) \sin ^{2}\left[\frac{\varepsilon_{1}+\varepsilon_{2}}{2} \tau\right] \\
& I_{s}(\tau)=\int_{q_{r}}^{1} d q q^{2}\left(1+\frac{q^{2}+m_{1} m_{2}}{\varepsilon_{1} \varepsilon_{2}}\right) \sin ^{2}\left[\frac{\varepsilon_{1}-\varepsilon_{2}}{2} \tau\right]
\end{aligned}
$$

We have separated the contributions from the fast $\left(I_{f}(\tau)\right)$ and slow $\left(I_{s}(\tau)\right)$ time scales as discussed in section (IIE) above. In particular, as discussed above the term that oscillates with the $\operatorname{sum} \varepsilon_{1}+\varepsilon_{2}$ is a consequence of the 
overlap between particles and antiparticles. The pre-factors that multiply the sine functions in equations (III.27III.28) arise from the overlap between particle-antiparticle spinors in (III.27) and particle-particle, anti-particle-anti-particle spinors in (III.28). The overlap between particle and antiparticle spinors is non-vanishing for different masses. Similar contributions from the overlap between particle and antiparticle states of different masses have been found in the studies of refs. 40, 41, 42].

Since the mass eigenstates are almost degenerate or alternatively for any values of the masses in the relativistic limit we find

$$
\begin{aligned}
\frac{q^{2}+m_{1} m_{2}}{\varepsilon_{1} \varepsilon_{2}} & =1-\frac{\bar{m}^{2} q^{2}}{\bar{\varepsilon}^{4}}\left(\frac{\Delta M^{2}}{4 \bar{M}^{2}}\right)^{2}+\mathcal{O}\left(\left(\frac{\Delta M^{2}}{4 \bar{M}^{2}}\right)^{4}\right) \\
\bar{\varepsilon} & =\sqrt{q^{2}+\bar{m}^{2}}
\end{aligned}
$$

with $\frac{\Delta M^{2}}{4 M^{2}} \sim 3 \times 10^{-4}$. Therefore the coefficient that results from the overlap between the particle and antiparticle spinors of different mass is given by

$$
1-\frac{q^{2}+m_{1} m_{2}}{\varepsilon_{1} \varepsilon_{2}}=\mathcal{O}\left(\frac{\Delta M^{2}}{4 \bar{M}^{2}} \frac{\bar{M}}{E(k)}\right)^{2}
$$

and the coefficient that results from the overlap between particle-particle or anti-particle-anti-particle of different masses is

$$
1+\frac{q^{2}+m_{1} m_{2}}{\varepsilon_{1} \varepsilon_{2}}=2+\mathcal{O}\left(\frac{\Delta M^{2}}{4 \bar{M}^{2}} \frac{\bar{M}}{E(k)}\right)^{2}
$$

where $E(k)$ is an energy scale.

Therefore the coefficient of the oscillatory term in $I_{f}(\tau)$ is a factor at least of order $\left(\frac{\Delta M^{2}}{4 M^{2}}\right)^{2} \sim 10^{-7}$ smaller than that of $I_{s}(\tau)$. Furthermore it is clear that the interference terms between particle and antiparticle average out on a time scale $t_{f} \lesssim 1 / \bar{M}$ whereas the particle-particle contributions evolve on a much slower time scale $t_{s} \sim M_{M} / \Delta M^{2} \gg t_{f}$.

However, despite the fact that the coefficients of the oscillatory terms in $I_{s}(\tau)$ and $I_{f}(\tau)$ differ by several orders of magnitude, the fact that the time evolution of $I_{s}(\tau)$ is much slower allows for a time scale within which both contributions are comparable. This can be gleaned from the following argument.

The integrals for $I_{s}(\tau)$ and $I_{f}(\tau)$ are dominated by the region $q \sim 1$. Consider an intermediate time scale so that the argument of the oscillatory function in $I_{f}(\tau)$ is of order one, but the argument of the oscillatory function in $I_{s}(\tau)$ is $\ll 1$. The contribution to the integral in $I_{f}(\tau)$ is of order $\bar{m}^{2}\left(\frac{\Delta m^{2}}{4 \bar{m}^{2}}\right)^{2}$ while the contribution to the integral $I_{s}(\tau)$ is of order $2\left(\delta m^{2} \tau^{2}\right)$. Therefore, it is clear that even when the prefactor of its oscillatory term is small, the integrand of $I_{f}(\tau)$ will be larger than that of $I_{s}(\tau)$ in the time domain during which

$$
\bar{m}^{2}\left(\frac{\delta m^{2}}{4 \bar{m}^{2}}\right)^{2}>\left(\delta m^{2} \tau\right)^{2} \Longrightarrow \tau \lesssim 1 / \bar{m}
$$

In the opposite limit, for $\tau>>1 / \bar{m}$ the dynamics is completely dominated by $I_{s}(\tau)$.

Fig. (11) below displays the early time evolution of $I_{s}(\tau)$ and $I_{f}(\tau)$ for $0 \leq \tau \lesssim 1 / \bar{m}$. It is clear from this figure that $I_{f}(\tau)$ averages out to its asymptotic value on a short time scale $\tau \sim 1\left(t \sim 1 / k_{F}\right)$ and that $I_{s}(\tau)$ begins to dominate the dynamics on time scales $\tau \gtrsim 1 / \bar{m}$ as discussed above. In the case of Fig.(1), with $k_{F}^{e}>>\bar{M}$ the time scale of averaging is $t \sim 1 / k_{F}^{e}$, but for $k_{F}<<\bar{M}$ it would be of order $1 / \bar{M}$.

In terms of dimensionful quantities the inequality obtained in eqn. (III.32) above translates into $t<1 / \bar{M}$. With the current estimate $\bar{M} \sim 0.25 \mathrm{eV}$ the analysis above suggests that the particle-antiparticle interference is dynamically relevant during time scales $t \lesssim 10^{-15} s$ although this time scale is comparable to the expansion time scale at the time of the electroweak phase transition, it is far shorter than the time scales relevant either for primordial nucleosynthesis or for dynamical processes during the collapse of supernovae or neutron star cooling.

While the behavior of $I_{s}(\tau)$ and $I_{f}(\tau)$ as a function of $\tau$ must in general be studied numerically, the long time limit can be extracted analytically.

The asymptotic long time behavior of $I_{s}(\tau)$ and $I_{f}(\tau)$ is determined by the end points of their integrands, in particular for momenta near the Fermi surface. Two relevant cases yield the following results 


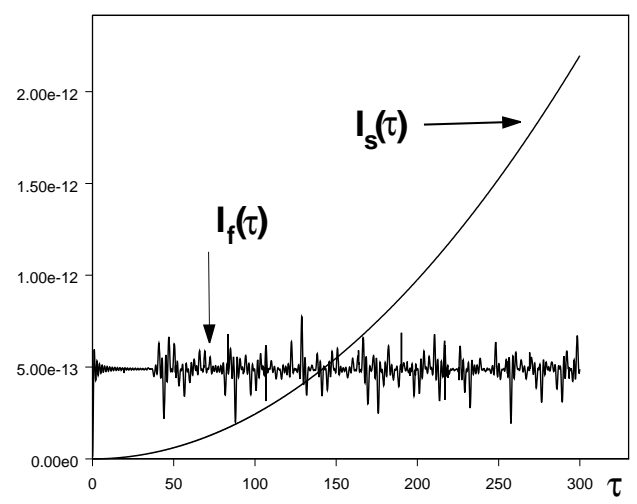

FIG. 1: $I_{s}(\tau)$ and $I_{s}(\tau)$ for $k_{F}^{e}=100 \mathrm{eV} ; k_{F}^{\mu}=0 ; \bar{M}=0.25 \mathrm{eV} ; \Delta M^{2} \simeq 7 \times 10^{-5}(\mathrm{eV})^{2}$ vs. $\tau$. For these values $1 / \bar{m}=400$.

- Relativistic Limit: $\max \left(k_{F}^{e}, k_{F}^{\mu}\right) \gg M_{1}, M_{2}$

$$
\begin{aligned}
& I_{s}(\tau)=\frac{1}{2} \int_{q_{r}}^{1} d q q^{2}\left(1+\frac{q^{2}+m_{1} m_{2}}{\varepsilon_{1} \varepsilon_{2}}\right)+\frac{2}{\delta m^{2} \tau}\left\{\sin \left(\frac{\delta m^{2}}{2} \tau\right)-q_{r}^{4} \sin \left(\frac{\delta m^{2} \tau}{2 q_{r}}\right)\right\}+\mathcal{O}\left(\frac{1}{\left(\delta m^{2} \tau\right)^{2}}\right)( \\
& I_{f}(\tau)=\frac{1}{2} \int_{q_{r}}^{1} d q q^{2}\left(1-\frac{q^{2}+m_{1} m_{2}}{\varepsilon_{1} \varepsilon_{2}}\right)-\frac{1}{8 \tau}\left(m_{1}-m_{2}\right)^{2}\left[\sin (2 \tau)-\sin 2 q_{r} \tau\right]+\mathcal{O}\left(\frac{1}{\tau^{2}}\right)
\end{aligned}
$$

where $\delta m^{2}$ is defined by equation (III.21) along with the other dimensionless variables.

- Non-Relativistic limit: $k_{F}^{e}, k_{F}^{\mu} \ll M_{1}, M_{2}$

$$
\begin{aligned}
I_{s}(\tau)= & \frac{1}{2} \int_{q_{r}}^{1} d q q^{2}\left(1+\frac{q^{2}+m_{1} m_{2}}{\varepsilon_{1} \varepsilon_{2}}\right)+\frac{m_{1} m_{2}}{\left(m_{1}-m_{2}\right) \tau}\left\{\sin \left[\left(m_{1}-m_{2}\right)\left(1-\frac{1}{2 m_{1} m_{2}}\right) \tau\right]-\right. \\
& \left.q_{r} \sin \left[\left(m_{1}-m_{2}\right)\left(1-\frac{q_{r}^{2}}{2 m_{1} m_{2}}\right) \tau\right]\right\}+\mathcal{O}\left(\frac{1}{\tau^{\frac{3}{2}}}\right) ; \text { for } \frac{\left(m_{1}-m_{2}\right) \tau}{m_{1} m_{2}} \gg 1 \\
I_{s}(\tau)= & \frac{1}{2} \int_{q_{r}}^{1} d q q^{2}\left(1+\frac{q^{2}+m_{1} m_{2}}{\varepsilon_{1} \varepsilon_{2}}\right)\left[1-\cos \left[\left(m_{1}-m_{2}\right) \tau\right]\right] \quad ; \text { for } \frac{\left(m_{1}-m_{2}\right) \tau}{m_{1} m_{2}} \ll 1 \\
I_{f}(\tau)= & \frac{1}{2} \int_{q_{r}}^{1} d q q^{2}\left(1-\frac{q^{2}+m_{1} m_{2}}{\varepsilon_{1} \varepsilon_{2}}\right)-\frac{\left(m_{1}-m_{2}\right)^{2}}{m_{1} m_{2}\left(m_{1}+m_{2}\right) \tau}\left\{\sin \left[\left(m_{1}+m_{2}\right)\left(1+\frac{1}{2 m_{1} m_{2}}\right) \tau\right]-\right. \\
& \left.q_{r}^{3} \sin \left[\left(m_{1}+m_{2}\right)\left(1+\frac{q_{r}^{2}}{2 m_{1} m_{2}}\right) \tau\right]\right\}+\mathcal{O}\left(\frac{1}{\tau^{2}}\right) ; \text { for } \frac{\left(m_{1}+m_{2}\right) \tau}{m_{1} m_{2}} \gg 1 \\
I_{f}(\tau)= & \frac{1}{2} \int_{q_{r}}^{1} d q q^{2}\left(1-\frac{q^{2}+m_{1} m_{2}}{\varepsilon_{1} \varepsilon_{2}}\right)\left[1-\cos \left[\left(m_{1}+m_{2}\right) \tau\right]\right] \quad ; \text { for } \frac{\left(m_{1}+m_{2}\right) \tau}{m_{1} m_{2}} \ll 1
\end{aligned}
$$

In both cases, the flavor asymmetry density at asymptotically long time is given by

$$
\frac{1}{V}\left(q_{e}(t)-q_{\mu}(t)\right) \rightarrow\left[\mathcal{N}^{(e)}-\mathcal{N}^{(\mu)}\right] \cos ^{2}(2 \theta)+\mathcal{O}(1 / t)
$$

The power law fall-off is a consequence of dephasing between different flavor modes that are not Pauli blocked. Fig.(2) displays the slow contribution $I_{s}(\tau)$ and its asymptotic limit given by eqn. (III.33) in the relativistic case. 

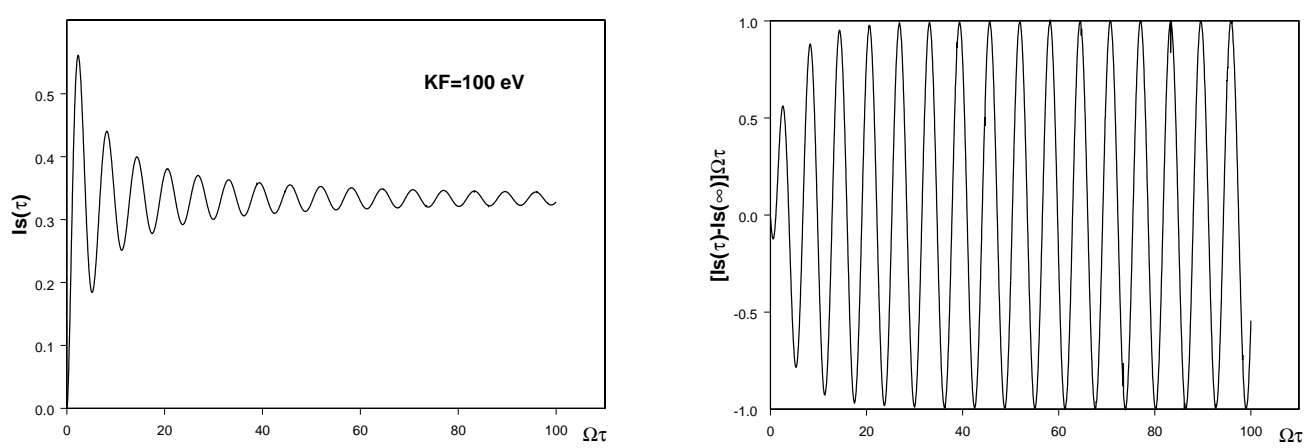

FIG. 2: $I_{s}(\tau)$ and $\left[I_{s}(\tau)-I_{s}(\infty)\right] \times(\Omega \tau)$ for $k_{F}^{e}=100 \mathrm{eV} ; k_{F}^{\mu}=0 ; \bar{M}=0.25 \mathrm{eV} ; \Delta M^{2} \simeq 7 \times 10^{-5}(\mathrm{eV})^{2}$ vs. $\Omega \tau$, with $\Omega=\delta m^{2}=7 \times 10^{-9}$.

\section{DISTRIBUTION FUNCTIONS OF NEUTRINOS AND ANTINEUTRINOS}

The distribution functions are given by equations (II.66)-(II.77) for which after lengthy but straightforward algebra we find the following expressions

$$
\begin{aligned}
I^{(e)}(k, t)=n^{(e)}(k)-2 n^{(e)}(k) \mathcal{A}(k, t)-\frac{k^{2}}{\omega_{e}^{2}(k)}\left[n^{(e)}(k)-\left(1-\bar{n}^{(e)}(k)\right)\right] \mathcal{B}(k, t) \\
J^{(e)}(k, t)=\left[\frac{C^{2} S^{2}\left(M_{1}-M_{2}\right)^{2}+M_{1} M_{2}+k^{2}}{\omega_{e}(k) \omega_{\mu}(k)}\left(n^{(\mu)}(k)-\left[1-\bar{n}^{(\mu)}(k)\right]\right)+\left(n^{(\mu)}(k)+\left[1-\bar{n}^{(\mu)}(k)\right]\right)\right] \mathcal{A}(k, t) \\
-\frac{k^{2}}{\omega_{e}(k) \omega_{\mu}(k)}\left[n^{(\mu)}(k)-\left(1-\bar{n}^{(\mu)}(k)\right)\right] \mathcal{C}(k, t)
\end{aligned}
$$

where $n^{(e)}(k)$ and $\bar{n}^{(e)}(k)$ are the initial distribution functions given by eqn. (II.20) and

$$
\begin{aligned}
\mathcal{A}(k, t)= & C^{2} S^{2}\left[\left(1-\frac{k^{2}+M_{1} M_{2}}{E_{1}(k) E_{2}(k)}\right) \sin ^{2}\left(\frac{E_{1}(k)+E_{2}(k)}{2} t\right)+\right. \\
& \left.\left(1+\frac{k^{2}+M_{1} M_{2}}{E_{1}(k) E_{2}(k)}\right) \sin ^{2}\left(\frac{E_{1}(k)-E_{2}(k)}{2} t\right)\right] \\
\mathcal{B}(k, t)= & C^{4} S^{4}\left(M_{1}-M_{2}\right)^{2}\left[\frac{1}{E_{1}(k)} \sin \left(E_{1}(k) t\right)-\frac{1}{E_{2}(k)} \sin \left(E_{2}(k) t\right)\right]^{2} \\
\mathcal{C}(k, t)= & C^{2} S^{2}\left(M_{1}-M_{2}\right)^{2}\left[\frac{C^{2} S^{2}}{E_{1}^{2}(k)} \sin ^{2}\left(E_{1}(k) t\right)+\frac{C^{2} S^{2}}{E_{2}^{2}(k)} \sin ^{2}\left(E_{2}(k) t\right)\right. \\
& \left.-\frac{2 C^{2} S^{2}-1}{E_{1}(k) E_{2}(k)} \sin \left(E_{1}(k) t\right) \sin \left(E_{2}(k) t\right)\right] .
\end{aligned}
$$

The expressions for $\bar{I}^{(e)}(k, t)$ and $\bar{J}^{(e)}(k, t)$ are obtained from those for $I^{(e)}(k, t)$ and $J^{(e)}(k, t)$ above by the replacement

$$
n^{(e)}(k) \longleftrightarrow\left[1-\bar{n}^{(e)}(k)\right] ; n^{(\mu)}(k) \longleftrightarrow\left[1-\bar{n}^{(\mu)}(k)\right]
$$

Finally the expressions for $I^{(\mu)}(k, t) ; \bar{I}^{(\mu)}(k, t) ; J^{(\mu)}(k, t) ; \bar{J}^{(\mu)}(k, t)$ are obtained from those for the electron neutrino by the replacement

$$
n^{(e)}(k) \longleftrightarrow n^{(\mu)}(k) ; \bar{n}^{(e)}(k) \longleftrightarrow \bar{n}^{(\mu)}(k) ; \omega_{e}(k) \longleftrightarrow \omega_{\mu}(k) ; C^{2} \longleftrightarrow S^{2}
$$


These dynamical factors $\mathcal{A}(k, t) ; \mathcal{B}(k, t) ; \mathcal{C}(k, t)$ are determined by the time evolution while their pre-factors in the expressions for the distribution functions are determined by the initial state. The dynamical factors clearly reveal again the different time scales. Terms that feature the contributions $e^{ \pm 2 i E_{(1,2)} t} ; e^{ \pm i\left(E_{1}+E_{2}\right) t}$ arise from particleantiparticle interference and their contribution is proportional to $\left(\Delta M^{2} / \bar{M}^{2}\right)$ and those that feature $e^{ \pm i\left(E_{1}-E_{2}\right) t}$ arise from particle-particle (or anti-particle- anti-particle) interference. We can find the asymptotic distribution functions at long time by averaging the oscillatory terms over a time scale longer than the longest scale $\sim \bar{M} / \Delta M^{2}$. This time averaging procedure leads to

$$
\begin{aligned}
& \overline{\mathcal{A}(k, t)}=C^{2} S^{2} \\
& \overline{\mathcal{B}(k, t)}=\overline{\mathcal{C}(k, t)}=\frac{1}{2} C^{4} S^{4}\left(M_{1}-M_{2}\right)^{2}\left[\frac{1}{E_{1}^{2}(k)}+\frac{1}{E_{2}^{2}(k)}\right]
\end{aligned}
$$

The above expressions are exact and therefore valid for any value of the neutrino masses $M_{1}, M_{2}$. However, the most recent compilation 10, 45] of data suggests that in the two flavor case the mass eigenstates are almost degenerate with $\Delta M^{2} \sim 7 \times 10^{-5}(\mathrm{eV})^{2}$ and the most recent cosmological constraint from WMAP [46] suggests that the average value of the mass $\bar{M}$ is $\lesssim 0.25(\mathrm{eV})$. In terms of the $\bar{M}$ and $\Delta M^{2}$ introduced in eqn. (III.19), we find

$$
m_{e}=\bar{M}\left[1+\frac{\Delta M^{2}}{4 \bar{M}^{2}} \cos (2 \theta)\right] ; m_{\mu}=\bar{M}\left[1-\frac{\Delta M^{2}}{4 \bar{M}^{2}} \cos (2 \theta)\right]
$$

In terms of the small ratio $\Delta M^{2} / \bar{M}^{2} \sim 10^{-3}$ we find the average of the distribution functions over the longest time scale to be given by

$$
\begin{aligned}
& n_{a v}^{(e)}(k)=n^{(e)}(k)-2 C^{2} S^{2}\left(n^{(e)}(k)-n^{(\mu)}(k)\right)-\mathcal{R}\left[k, n^{(\alpha)}, \bar{n}^{(\alpha)}\right] \\
& \bar{n}_{a v}^{(e)}(k)=\bar{n}^{(e)}(k)-2 C^{2} S^{2}\left(\bar{n}^{(e)}(k)-\bar{n}^{(\mu)}(k)\right)-\mathcal{R}\left[k, n^{(\alpha)}, \bar{n}^{(\alpha)}\right] \\
& n_{a v}^{(\mu)}(k)=n^{(\mu)}(k)+2 C^{2} S^{2}\left(n^{(e)}(k)-n^{(\mu)}(k)\right)-\mathcal{R}\left[k, n^{(\alpha)}, \bar{n}^{(\alpha)}\right] \\
& \bar{n}_{a v}^{(\mu)}(k)=\bar{n}^{(\mu)}(k)+2 C^{2} S^{2}\left(\bar{n}^{(e)}(k)-\bar{n}^{(\mu)}(k)\right)-\mathcal{R}\left[k, n^{(\alpha)}, \bar{n}^{(\alpha)}\right]
\end{aligned}
$$

with

$$
\begin{aligned}
\mathcal{R}\left[k, n^{(\alpha)}, \bar{n}^{(\alpha)}\right]= & \frac{k^{2} \bar{M}^{2}}{\bar{E}^{4}(k)}\left(\frac{\Delta M^{2}}{4 \bar{M}^{2}}\right)^{2} C^{2} S^{2}\left[4 C^{2} S^{2}\left(n^{(e)}(k)+\bar{n}^{(e)}(k)-1\right)+\left(n^{(\mu)}(k)+\bar{n}^{(\mu)}(k)-1\right)\right] \\
& +\mathcal{O}\left(\left(\frac{\Delta M^{2}}{4 \bar{M}^{2}}\right)^{3}\right) \\
\bar{E}(k)=\sqrt{k^{2}+\bar{M}^{2}} &
\end{aligned}
$$

The term $\mathcal{R}\left[k, n^{(\alpha)}, \bar{n}^{(\alpha)}\right]$ arises from the overlap between particle and antiparticle spinors which features the small quantity $\left(\frac{\Delta M^{2}}{4 M^{2}}\right)$.

Flavor pair production and normal ordering:

The expressions IV.12 IV.15) with that for the corrections given by eqn. (IV.16) point out an important and subtle aspect of the dynamics of mixing. Consider that the initial density matrix is the flavor vacuum, namely set $n^{(e)}(k)=\bar{n}^{(e)}(k)=n^{(\mu)}(k)=\bar{n}^{(\mu)}(k)=0$. The asymptotic limit of the distribution functions (IV.12 IV.15) is given to lowest non trivial order in the ratio $\Delta M^{2} / \bar{M}^{2}$ by

$$
\begin{aligned}
n^{(e)}(k, \infty)=\bar{n}^{(e)}(k, \infty)=n^{(\mu)}(k, \infty)=\bar{n}^{(\mu)}(k, \infty)= & \frac{k^{2} \bar{M}^{2}}{4 \bar{E}^{4}(k)}\left(\frac{\Delta M^{2}}{4 \bar{M}^{2}}\right)^{2} \sin ^{2}(2 \theta)\left(1+\sin ^{2}(2 \theta)\right)+ \\
& \mathcal{O}\left(\left(\frac{\Delta M^{2}}{4 \bar{M}^{2}}\right)^{3}\right)
\end{aligned}
$$


This result clearly indicates that the time evolution results in the creation of particle-antiparticle pairs of electron and muon neutrinos. This is of course a consequence of the non-vanishing overlap between positive and negative energy spinors which results in that a destruction operator for flavor neutrinos develops a component corresponding to a creation operator of antineutrinos during time evolution, and viceversa. In leading order in the degeneracy, the typical momentum of the pair created is $k \sim \bar{M}$ therefore these are typically low momentum pairs of flavor neutrinos.

Furthermore a remarkable aspect of this pair production process via neutrino mixing is that the distribution function of the produced particles falls off very slowly at high energies, namely $n_{\text {prod }}(k, \infty) \propto 1 / k^{2}$. As a result there is a divergent number of pairs produced as a consequence of mixing and time evolution. Since the particles and antiparticles are produced in pairs, the flavor charge vanishes, but the individual distribution functions feature a contribution from the pair production process. A normal ordering prescription must be appended to subtract the infinite number of particles created, however unlike normal ordering in the usual free field theory, which subtracts a constant, in the case of mixing such normal ordering requires a subtraction of a distribution function.

This is a novel and subtle phenomenon, flavor pair production which is a direct many particle consequence of mixing and oscillations. Since this phenomenon is a consequence of the interference between particle and antiparticle states is suppressed by the small quantity $\left(\Delta M^{2} / \bar{M}\right)^{2}$.

Regardless of whether this phenomenon of flavor pair production has any bearing on the cosmology and or astrophysics of neutrinos, it is a genuine many body aspect inherent to the field theory of neutrino mixing that deserves to be studied in its own right as a fundamental aspect of the field theory of mixing.

Off-diagonal densities: Even when the initial density matrix is diagonal in the flavor basis and therefore there are no off-diagonal initial correlations, these develop upon time evolution as a consequence of flavor mixing. Following the same steps described above for the distribution functions, we find the off-diagonal density to be given by the following expression

$$
\begin{aligned}
<\nu_{e}^{\dagger}(\vec{k}, t) \nu_{\mu}(\vec{k}, t)>= & 2 \frac{C^{2}-S^{2}}{C S}\left[\left(n^{(e)}(k)-\bar{n}^{(e)}(k)\right)-\left(n^{(\mu)}(k)-\bar{n}^{(\mu)}(k)\right)\right] \mathcal{A}(k, t) \\
+ & i C S \sin \left[\left(E_{1}(k)-E_{2}(k)\right) t\right] \times \\
& \left\{\frac{1}{\omega_{e}(k)}\left[n^{(e)}(k)-\left(1-\bar{n}^{(e)}(k)\right)\right]\left[\left(E_{1}(k)+E_{2}(k)\right)-\left(M_{1}-M_{2}\right)\left(\frac{S^{2} M_{1}}{E_{1}(k)}-\frac{C^{2} M_{2}}{E_{2}(k)}\right)\right]\right. \\
& \left.\quad-\frac{1}{\omega_{\mu}(k)}\left[n^{(\mu)}(k)-\left(1-\bar{n}^{(\mu)}(k)\right)\right]\left[\left(E_{1}(k)+E_{2}(k)\right)-\left(M_{1}-M_{2}\right)\left(\frac{C^{2} M_{1}}{E_{1}(k)}-\frac{S^{2} M_{2}}{E_{2}(k)}\right)\right]\right\} \\
+ & i C S \sin \left[\left(E_{1}(k)+E_{2}(k)\right) t\right] \times \\
& \left\{\frac{1}{\omega_{e}(k)}\left[n^{(e)}(k)-\left(1-\bar{n}^{(e)}(k)\right)\right]\left[\left(E_{1}(k)-E_{2}(k)\right)-\left(M_{1}-M_{2}\right)\left(\frac{S^{2} M_{1}}{E_{1}(k)}+\frac{C^{2} M_{2}}{E_{2}(k)}\right)\right]\right. \\
& \left.-\frac{1}{\omega_{\mu}(k)}\left[n^{(\mu)}(k)-\left(1-\bar{n}^{(\mu)}(k)\right)\right]\left[\left(E_{1}(k)-E_{2}(k)\right)-\left(M_{1}-M_{2}\right)\left(\frac{C^{2} M_{1}}{E_{1}(k)}+\frac{S^{2} M_{2}}{E_{2}(k)}\right)\right]\right\}
\end{aligned}
$$

with $\mathcal{A}(k, t)$ given by eqn. (IV.3). The expressions for the distribution functions and the off-diagonal density can be simplified by expanding the coefficients of the oscillatory functions up to leading order in the small quantity $\left(\Delta M^{2} / \bar{M}^{2}\right)$. We find

$$
n^{(e)}(k, t)=n^{(e)}(k)-\left(n^{(e)}(k)-n^{(\mu)}(k)\right) 2 C^{2} S^{2}\left[1-\cos \left[\left(E_{1}(k)-E_{2}(k)\right) t\right]\right]+\mathcal{O}\left(\frac{\Delta M^{2}}{4 \bar{M}^{2}}\right)^{2}
$$

the other distribution functions may be found from the expression above by the replacements in eqns. (IV.7IV.8). Their time averages over the long time scale coincides with the leading expressions in eqns. (IV.12 IV.15). The off-diagonal density simplifies to the following expression 


$$
\begin{aligned}
<\nu_{e}^{\dagger}(\vec{k}, t) \nu_{\mu}(\vec{k}, t)>= & -2 S C\left\{2\left(C^{2}-S^{2}\right)\left[\left(n^{(e)}(k)-\bar{n}^{(e)}(k)\right)-\left(n^{(\mu)}(k)-\bar{n}^{(\mu)}(k)\right)\right] \sin ^{2}\left[\left(E_{1}(k)-E_{2}(k)\right) \frac{t}{2}\right]\right. \\
& \left.-i\left[\left(n^{(e)}(k)+\bar{n}^{(e)}(k)\right)-\left(n^{(\mu)}(k)+\bar{n}^{(\mu)}(k)\right)\right] \sin \left[\left(E_{1}(k)-E_{2}(k)\right) t\right]\right\} \\
& +\mathcal{O}\left(\frac{\Delta M^{2}}{4 \bar{M}^{2}}\right)^{2}
\end{aligned}
$$

The terms of $\mathcal{O}\left(\frac{\Delta M^{2}}{4 M^{2}}\right)^{2}$ again involve terms that oscillate with the sum of the frequencies corresponding to particleantiparticle interference as well as terms that oscillate with the difference of the frequencies arising from the overlap of the particle (or antiparticle) spinor wavefunctions for different masses. The analysis that was presented for the same type of contributions in $I_{s}(\tau) ; I_{f}(\tau)$ above highlight that the particle-antiparticle interference becomes subdominant on time scales $t>1 / \bar{M}$. Hence the first terms $\mathcal{O}\left(\frac{\Delta M^{2}}{4 M^{2}}\right)^{0}$ in the approximations (IV.20 IV.21) determine the dynamics of the distribution functions and the off-diagonal correlator in leading order in the small ratio $\frac{\Delta M^{2}}{4 M^{2}}$ for $t>>1 / \bar{M}$.

\section{A. Equilibrated gas of mass eigenstates}

Although we have focused on the case in which the initial density matrix is diagonal in the flavor basis, for completeness we now study the case in which the initial density matrix describes an ensemble of mass eigenstates in equilibrium. Therefore this initial density matrix is diagonal in the mass basis and commutes with the Hamiltonian. This situation thus describes a state of equilibrium in which the occupation numbers do not evolve in time (in the non-interacting theory). In this case we find

$$
\begin{aligned}
<\left[\bar{\psi}_{i}(\vec{k}, 0)\right]_{r}\left[\psi_{i}(\vec{k}, 0)\right]_{s}> & =n^{(i)}(k)\left(\frac{\not i_{i}+M_{i}}{2 E_{i}(k)}\right)_{s r}+\left(1-\bar{n}^{(i)}(k)\right)\left[\gamma^{0} \frac{\not k_{i}-M_{i}}{2 E_{i}(k)} \gamma^{0}\right]_{s r} \equiv\left[N_{i}(\vec{k})\right]_{s r} \\
\not k_{i} & =\gamma^{0} E_{i}(\vec{k})-\vec{\gamma} \cdot \vec{k}
\end{aligned}
$$

where $n^{(i)}(k)$ are the occupation numbers of mass eigenstates assumed to depend only on the energy. Just as we did in our previous analysis it proves convenient to write the above correlator in the following form

$$
\begin{aligned}
N_{i}(\vec{k}) & =Q_{i}+\tilde{M}_{i} \\
Q_{i} & =\gamma^{0} Q_{i}^{0}-\vec{\gamma} \cdot \vec{Q}_{i}, \\
Q_{i}^{0} & =\frac{1}{2}\left[n^{(i)}(k)+\left(1-\bar{n}^{(i)}(k)\right)\right], \\
\vec{Q}_{i} & =\frac{\vec{k}}{2 E_{i}(k)}\left[n^{(i)}(k)-\left(1-\bar{n}^{(i)}(k)\right)\right], \\
\tilde{M}_{i} & =\frac{M_{i}}{2 E_{i}(k)}\left[n^{(i)}(k)-\left(1-\bar{n}^{(i)}(k)\right)\right] .
\end{aligned}
$$

Since the density matrix commutes with the full Hamiltonian, the distribution functions of the flavor eigenstates do not depend on time. Following the procedure detailed above we find the following results

$$
\begin{aligned}
n^{(e)}(k)= & \frac{C^{2}}{2}\left[\left(1+\frac{k^{2}+m_{e} M_{1}}{\omega_{e}(k) E_{1}(k)}\right) n^{(1)}(k)+\left(1-\frac{k^{2}+m_{e} M_{1}}{\omega_{e}(k) E_{1}(k)}\right)\left(1-\bar{n}^{(1)}(k)\right)\right] \\
& +\frac{S^{2}}{2}\left[\left(1+\frac{k^{2}+m_{e} M_{2}}{\omega_{e}(k) E_{2}(k)}\right) n^{(2)}(k)+\left(1-\frac{k^{2}+m_{e} M_{2}}{\omega_{e}(k) E_{2}(k)}\right)\left(1-\bar{n}^{(2)}(k)\right)\right] \\
\bar{n}^{(e)}(k)= & 1-\frac{C^{2}}{2}\left[\left(1-\frac{k^{2}+m_{e} M_{1}}{\omega_{e}(k) E_{1}(k)}\right) n^{(1)}(k)+\left(1+\frac{k^{2}+m_{e} M_{1}}{\omega_{e}(k) E_{1}(k)}\right)\left(1-\bar{n}^{(1)}(k)\right)\right] \\
& -\frac{S^{2}}{2}\left[\left(1-\frac{k^{2}+m_{e} M_{2}}{\omega_{e}(k) E_{2}(k)}\right) n^{(2)}(k)+\left(1+\frac{k^{2}+m_{e} M_{2}}{\omega_{e}(k) E_{2}(k)}\right)\left(1-\bar{n}^{(2)}(k)\right)\right]
\end{aligned}
$$


Using the relations given by eqn. (IV.11) we find to leading order in $\Delta M^{2} / \bar{M}^{2}$

$$
\begin{aligned}
& n^{(e)}(k)=C^{2} n^{(1)}(k)+S^{2} n^{(2)}(k)+\mathcal{O}\left(\frac{\Delta M^{2}}{4 \bar{M}^{2}}\right)^{2} \\
& n^{(\mu)}(k)=S^{2} n^{(1)}(k)+C^{2} n^{(2)}(k)+\mathcal{O}\left(\frac{\Delta M^{2}}{4 \bar{M}^{2}}\right)^{2} \\
& \bar{n}^{(e)}(k)=C^{2} \bar{n}^{(1)}(k)+S^{2} \bar{n}^{(2)}(k)+\mathcal{O}\left(\frac{\Delta M^{2}}{4 \bar{M}^{2}}\right)^{2} \\
& \bar{n}^{(\mu)}(k)=S^{2} \bar{n}^{(1)}(k)+C^{2} \bar{n}^{(2)}(k)+\mathcal{O}\left(\frac{\Delta M^{2}}{4 \bar{M}^{2}}\right)^{2}
\end{aligned}
$$

\section{V. "EFFECTIVE" (FREE) FIELD THEORY DESCRIPTION:}

Let us summarize the lessons learned in the analysis of the previous section in order to establish a set of criteria with which to develop an effective description of the dynamics in the case in which the mass eigenstates are nearly degenerate as confirmed by the experimental situation or in the relativistic case.

- For nearly degenerate mass eigenstates there is a hierarchy of scales determined by i) $k_{F}$ or temperature (T), ii) the average mass $\bar{M}$ and iii) the mass difference $M_{1}-M_{2}$. The experimental situation seems to confirm the near degeneracy with $\left|M_{1}-M_{2}\right| \ll \bar{M}$, therefore at least two scales are widely separated. Furthermore if $k_{F}$ and or $T$ (temperature) are such that $k_{F} ; T \gg \bar{M}$ which describes a relativistic case, then all three scales are widely separated with the hierarchy $k_{F}, T \gg \bar{M} \gg\left|M_{1}-M_{2}\right|$. The dynamics studied above reveals all three scales.

- The time evolution of the distribution functions, flavor asymmetry and off-diagonal correlators all feature terms that oscillate with the frequencies $E_{1}(k)+E_{2}(k), 2 E_{1,2}(k)$, and also terms which oscillate with the difference $E_{1}(k)-E_{2}(k)$. The former arise from the interference between particle and antiparticle states of equal or different masses and determine the short time scales $t \lesssim 1 / \bar{M}$, while the latter arise from interference between particle states (or antiparticle states) of different masses and determine the long time scales $t \gtrsim \bar{M} / \Delta M^{2}$. The terms that oscillate with the fast time scales average out on these fast scales and their coefficients are of order $\Delta M^{2} / \bar{M}^{2}$ and hence small in the nearly degenerate case. These coefficients result from the overlap between positive and negative energy spinors of slightly different masses. The coefficients of the terms that oscillate on the long time scale are of $\mathcal{O}(1)$ and result from the overlap between positive energy spinors (or between negative energy spinors) of different masses.

- The contributions to the distribution functions and off-diagonal correlators from the terms with fast and slow oscillations are comparable within the short time scale $t \lesssim 1 / \bar{M}$ but for times longer than this scale the contributions from the terms with fast oscillations are suppressed with respect to those with slow oscillations at least by $\mathcal{O}\left(\frac{\Delta M^{2}}{4 M^{2}}\right)^{2}$.

We seek to obtain a description of the oscillation dynamics on scales much larger than $1 / \bar{M}$ when the contribution from the fast oscillations have averaged out to quantities that are proportional to powers of the small ratio $\frac{\Delta M^{2}}{4 M^{2}}$ and can therefore be neglected in the nearly degenerate case.

In the nearly degenerate case $\Delta M^{2} / \bar{M}^{2} \ll 1$ the masses $m_{e}, m_{\mu}, M_{1}, M_{2} \sim \bar{M}$ (see eqns. (IV.11) (II.20) ), thus in order to isolate the leading order terms as well as to understand corrections in the degeneracy parameter $\Delta M^{2} / \bar{M}^{2}$ it proves convenient to expand the positive and negative energy spinors in terms of this small parameter. A straightforward computation in the standard Dirac representation of the Dirac gamma matrices leads to the following result for the flavor positive and negative energy spinors (see eqn. (II.6)

$$
\begin{aligned}
U_{\vec{k}, \lambda}^{(\alpha)} & =\left[1 \pm \frac{\Delta M^{2}}{4 \bar{M}^{2}} \frac{\bar{M}}{\bar{E}(k)} \cos (2 \theta)\left(\frac{\gamma^{0} \bar{E}(k)-\bar{M}}{2 \bar{E}(k)}\right)+\mathcal{O}\left(\frac{\Delta M^{2}}{4 \bar{M}^{2}}\right)^{2}\right] \mathcal{U}_{\vec{k}, \lambda} \\
V_{-\vec{k}, \lambda}^{(\alpha)} & =\left[1 \mp \frac{\Delta M^{2}}{4 \bar{M}^{2}} \frac{\bar{M}}{\bar{E}(k)} \cos (2 \theta)\left(\frac{\gamma^{0} \bar{E}(k)+\bar{M}}{2 \bar{E}(k)}\right)+\mathcal{O}\left(\frac{\Delta M^{2}}{4 \bar{M}^{2}}\right)^{2}\right] \mathcal{V}_{\vec{k}, \lambda}
\end{aligned}
$$


with

$$
\bar{E}(k)=\sqrt{k^{2}+\bar{M}^{2}}
$$

and the upper sign corresponds to $\alpha=e$ and the lower sign to $\alpha=\mu$. The spinors $\mathcal{U}_{\vec{k}, \lambda}, \mathcal{V}_{\vec{k}, \lambda}$ are positive and negative energy solutions respectively of the Dirac equation with mass $\bar{M}$ with unit normalization. Similarly for the positive and negative energy spinors associated with the mass eigenstates $F_{\vec{k}, \lambda}^{(i)} ; G_{-\vec{k}, \lambda}^{(i)}$ (see eqn. (II.10) ), we find

$$
\begin{aligned}
F_{\vec{k}, \lambda}^{(i)} & =\left[1 \pm \frac{\Delta M^{2}}{4 \bar{M}^{2}} \frac{\bar{M}}{\bar{E}(k)}\left(\frac{\gamma^{0} \bar{E}(k)-\bar{M}}{2 \bar{E}(k)}\right)+\mathcal{O}\left(\frac{\Delta M^{2}}{4 \bar{M}^{2}}\right)^{2}\right] \mathcal{U}_{\vec{k}, \lambda} \\
G_{-\vec{k}, \lambda}^{(i)} & =\left[1 \mp \frac{\Delta M^{2}}{4 \bar{M}^{2}} \frac{\bar{M}}{\bar{E}(k)}\left(\frac{\gamma^{0} \bar{E}(k)+\bar{M}}{2 \bar{E}(k)}\right)+\mathcal{O}\left(\frac{\Delta M^{2}}{4 \bar{M}^{2}}\right)^{2}\right] \mathcal{V}_{\vec{k}, \lambda}
\end{aligned}
$$

with the same spinors $\mathcal{U}_{\vec{k}, \lambda} ; \mathcal{V}_{\vec{k}, \lambda}$, where the upper sign corresponds to $i=1$ and the lower sign to $i=2$.

It is clear from the approximations (V.1) and (V.3) that the overlap between positive and negative energy spinors of different masses is $\mathcal{O}\left(\frac{\Delta M^{2}}{4 M^{2}}\right)^{2}$. For times much larger than the fast time scale, the corrections to the spinors are subdominant and can be neglected and the fields associated with the flavor and mass eigenstates are expanded as

$$
\begin{aligned}
\nu_{\alpha}(\vec{k}, t) & =\sum_{\lambda}\left(\alpha_{\vec{k}, \lambda}^{(\alpha)}(t) \mathcal{U}_{\vec{k}, \lambda}+\beta_{-\vec{k}, \lambda}^{(\alpha) \dagger}(t) \mathcal{V}_{-\vec{k}, \lambda}\right)+\mathcal{O}\left(\frac{\Delta M^{2}}{4 \bar{M}^{2}}\right) \\
\psi_{i}(\vec{k}, t) & =\sum_{\lambda}\left(a_{\vec{k}, \lambda}^{(i)} \mathcal{U}_{\vec{k}, \lambda} e^{-i E_{i}(k) t}+b_{-\vec{k}, \lambda}^{(i) \dagger} \mathcal{V}_{-\vec{k}, \lambda} e^{i E_{i}(k) t}\right)+\mathcal{O}\left(\frac{\Delta M^{2}}{4 \bar{M}^{2}}\right) .
\end{aligned}
$$

We can now find the relation between the creation and annihilation operators of flavor states and those of mass eigenstates by using eqn. (II.2), to leading order in the degeneracy parameter we find

$$
\begin{aligned}
\alpha_{\vec{k}, \lambda}^{(e)}(t) & =C a_{\vec{k}, \lambda}^{(1)} e^{-i E_{1}(k) t}+S a_{\vec{k}, \lambda}^{(2)} e^{-i E_{2}(k) t} \\
\alpha_{\vec{k}, \lambda}^{(\mu)}(t) & =C a_{\vec{k}, \lambda}^{(2)} e^{-i E_{2}(k) t}-S a_{\vec{k}, \lambda}^{(1)} e^{-i E_{1}(k) t}
\end{aligned}
$$

where we have neglected terms of $\mathcal{O}\left(\frac{\Delta M^{2}}{4 M^{2}}\right)$, and similar relations hold for the annihilation operators of the respective antiparticles $\beta_{\vec{k}, \lambda}^{(\alpha)}(t)$. It is clear that the approximations leading to the relations (V.6) and (V.7) are more generally valid not only in the nearly degenerate case but also in the relativistic case $k \gg M_{1,2}$ regardless of the value of the mass difference, since in this case the common spinors are those of massless Dirac fermions in all cases.

In this approximation, the evolution equation for the Heisenberg operators $\alpha_{\vec{k}, \lambda}^{(\alpha)}(t)$ does not follow directly from any Dirac equation, but can be obtained straightforwardly by taking time derivatives of these operators in eqns. (V.6 V.7) and using the relations V.6V.7 to re-write the result in terms of the operators themselves. In the leading order approximation particles and antiparticles do not mix since the overlap between the spinors $\mathcal{U}_{\vec{k}, \lambda}$ and $\mathcal{V}_{-\vec{k}, \lambda}$ vanishes (in free field theory) and a straightforward calculation leads to the following equations of motion

$$
i \frac{d}{d t}\left(\begin{array}{c}
\alpha_{\vec{k}, \lambda}^{(e)}(t) \\
\alpha_{\vec{k}, \lambda}^{(\mu)}(t)
\end{array}\right)=\left[\bar{E}(k)\left(\begin{array}{ll}
1 & 0 \\
0 & 1
\end{array}\right)-\Omega(k)\left(\begin{array}{cc}
-\cos (2 \theta) & \sin (2 \theta) \\
\sin (2 \theta) & \cos (2 \theta)
\end{array}\right)\right]\left(\begin{array}{c}
\alpha_{\vec{k}, \lambda}^{(e)}(t) \\
\alpha_{\vec{k}, \lambda}^{(\mu)}(t)
\end{array}\right)
$$

with

$$
\begin{aligned}
& \bar{E}(k)=\frac{1}{2}\left(E_{1}(k)+E_{2}(k)\right)=\sqrt{k^{2}+\bar{M}^{2}}+\mathcal{O}\left(\frac{\Delta M^{2}}{4 \bar{M}^{2}}\right) \\
& \Omega(k)=\frac{1}{2}\left(E_{1}(k)-E_{2}(k)\right)=\frac{\Delta M^{2}}{4 \bar{E}(k)}+\mathcal{O}\left(\frac{\Delta M^{2}}{4 \bar{M}^{2}}\right)
\end{aligned}
$$


and a similar equation of motion for the annihilation operators for flavor antiparticles $\beta_{\vec{k}, \lambda}^{(\alpha)}(t)$. These equations of motion look to be the familiar ones for neutrino oscillations [1]-[4, 12, 16, 22, 23], but these are equations for the Heisenberg field operators, rather than for the single particle wave-functions. Once the time evolution of the operators is found, we can find the time evolution of any multiparticle state. Furthermore the regime of validity of these equations is more general, they are valid either in the nearly degenerate case $\Delta M^{2} / \bar{M}^{2} \ll 1$ for any value of the momentum, or in the relativistic limit for arbitrary value of the masses provided that $k \gg M_{1}, M_{2}$.

Inverting the relation between the operators for flavor and mass states at the initial time, namely writing the operators $a_{\vec{k}, \lambda}^{(i)}$ in terms of $\alpha_{\vec{k}, \lambda}^{(\alpha)}(0)$ using eqns. (V.6

$$
\begin{aligned}
& \alpha_{\vec{k}, \lambda}^{(e)}(t)=\alpha_{\vec{k}, \lambda}^{(e)}(0)\left[C^{2} e^{-i E_{1}(k) t}+S^{2} e^{-i E_{2}(k) t}\right]+S C \alpha_{\vec{k}, \lambda}^{(\mu)}(0)\left[e^{-i E_{2}(k) t}-e^{-i E_{1}(k) t}\right] \\
& \alpha_{\vec{k}, \lambda}^{(\mu)}(t)=\alpha_{\vec{k}, \lambda}^{(\mu)}(0)\left[C^{2} e^{-i E_{2}(k) t}+S^{2} e^{-i E_{1}(k) t}\right]+S C \alpha_{\vec{k}, \lambda}^{(e)}(0)\left[e^{-i E_{2}(k) t}-e^{-i E_{1}(k) t}\right]
\end{aligned}
$$

For the antiparticle operators we find the same equations with $\alpha_{\vec{k}, \lambda}^{(\alpha)} \rightarrow \beta_{\vec{k}, \lambda}^{(\alpha)}$.

The Heisenberg field operators given by eqns. (V.11V.12) (and the equivalent for the antiparticle operators) are the solutions of the equations of motion (V.8).

The time evolution of the distribution functions in an initial density matrix that is diagonal in the flavor basis follows from a straightforward calculation using the above time evolution. We find

$$
\begin{aligned}
& n^{(e)}(k, t)=\left\langle\alpha_{\vec{k}, \lambda}^{(e) \dagger}(t) \alpha_{\vec{k}, \lambda}^{(e)}(t)\right\rangle=n^{(e)}(k)-\frac{1}{2} \sin ^{2}(2 \theta)\left(n^{(e)}(k)-n^{(\mu)}(k)\right)\left[1-\cos \left[\left(E_{1}(k)-E_{2}(k)\right) t\right]\right] \\
& n^{(\mu)}(k, t)=\left\langle\alpha_{\vec{k}, \lambda}^{(\mu) \dagger}(t) \alpha_{\vec{k}, \lambda}^{(\mu)}(t)\right\rangle=n^{(\mu)}(k)+\frac{1}{2} \sin ^{2}(2 \theta)\left(n^{(e)}(k)-n^{(\mu)}(k)\right)\left[1-\cos \left[\left(E_{1}(k)-E_{2}(k)\right) t\right]\right]
\end{aligned}
$$

The distribution functions for antiparticles to leading order is obtained from the above results by the replacements $n^{(\alpha)} \rightarrow \bar{n}^{(\alpha)}$. A straightforward calculation following the above steps leads to the result

$$
\begin{aligned}
<\nu_{e}^{\dagger}(\vec{k}, t) \nu_{\mu}(\vec{k}, t)>= & -\sin (2 \theta)\left\{2 \cos (2 \theta)\left[\left(n^{(e)}(k)-\bar{n}^{(e)}(k)\right)-\left(n^{(\mu)}(k)-\bar{n}^{(\mu)}(k)\right)\right] \sin ^{2}\left[\left(E_{1}(k)-E_{2}(k)\right) \frac{t}{2}\right]\right. \\
& \left.-i\left[\left(n^{(e)}(k)+\bar{n}^{(e)}(k)\right)-\left(n^{(\mu)}(k)+\bar{n}^{(\mu)}(k)\right)\right] \sin \left[\left(E_{1}(k)-E_{2}(k)\right) t\right]\right\}
\end{aligned}
$$

The results (V.13) and $(\nabla .15$ reproduce the leading order expressions found in the previous section, eqns. (IV.20IV.21). Thus this "effective" free field theory description reproduces the leading order results either in the nearly degenerate case $\Delta M^{2} \ll \bar{M}^{2}$ or in the relativistic case. Furthermore either the effective equations of motion (V.8) or alternatively the time evolution (V.11V.12) (and those for antiparticles) lead to a set of closed evolution equations for bilinears. These are most conveniently written by introducing a fiducial spin $\vec{S}=\left(S_{x}, S_{y}, S_{z}\right)$ with the following components

$$
\begin{aligned}
S_{x}(\vec{k}, \lambda ; t) & =i\left(\alpha_{\vec{k}, \lambda}^{(\mu) \dagger}(t) \alpha_{\vec{k}, \lambda}^{(e)}(t)-\alpha_{\vec{k}, \lambda}^{(e) \dagger}(t) \alpha_{\vec{k}, \lambda}^{(\mu)}(t)\right) \\
S_{y}(\vec{k}, \lambda ; t) & =\left(\alpha_{\vec{k}, \lambda}^{(\mu) \dagger}(t) \alpha_{\vec{k}, \lambda}^{(e)}(t)+\alpha_{\vec{k}, \lambda}^{(e) \dagger}(t) \alpha_{\vec{k}, \lambda}^{(\mu)}(t)\right) \\
S_{z}(\vec{k}, \lambda ; t) & =\left(\alpha_{\vec{k}, \lambda}^{(e) \dagger}(t) \alpha_{\vec{k}, \lambda}^{(e)}(t)-\alpha_{\vec{k}, \lambda}^{(\mu) \dagger}(t) \alpha_{\vec{k}, \lambda}^{(\mu)}(t)\right)
\end{aligned}
$$

and a fiducial magnetic field $\vec{B}=\left(B_{x}, B_{y}, B_{z}\right)$ with components

$$
\vec{B}(k)=2 \Omega(k)(0,-\sin (2 \theta), \cos (2 \theta))
$$

in terms of which the equations for the bilinears are akin to the Bloch equations for a spin $\vec{S}$ precessing in the magnetic field $\vec{B}$ namely

$$
\frac{d \vec{S}(\vec{k}, \lambda ; t)}{d t}=\vec{S}(\vec{k}, \lambda ; t) \times \vec{B}(k)
$$


The antiparticle operators obey independently a similar set of equations. To leading order in $\Delta M^{2} / \bar{M}^{2}$ there is no mixing between particles and antiparticles (suppressed by two powers of this small ratio), therefore the number of electron plus muon neutrinos is conserved independently of that for antineutrinos, namely

$$
\frac{d}{d t}\left(\alpha_{\vec{k}, \lambda}^{(e) \dagger}(t) \alpha_{\vec{k}, \lambda}^{(e)}(t)+\alpha_{\vec{k}, \lambda}^{(\mu) \dagger}(t) \alpha_{\vec{k}, \lambda}^{(\mu)}(t)\right)=0
$$

and similarly for the operators $\beta_{\vec{k}, \lambda}^{(\alpha)}$. The set of equations above, for Heisenberg operators is akin to the equations of motion for the "single particle" density matrix obtained in ref. [28], which are equivalent to those investigated in refs. $26,29,30,32,35]$.

In the study of synchronized oscillations [28, 30, 31, 35], a self-consistent Hartree-Fock approximation is introduced which leads to a Bloch equation like (V.20) but where the magnetic field $\vec{B}$ acquires a correction from the selfconsistent Hartree terms which arise from forward scattering off neutrinos in the medium.

This effective formulation neglects the dynamics of flavor pair production discussed above since such phenomenon is suppressed by two powers of the small ratio $\Delta M^{2} / \bar{M}^{2}$.

\section{A. Propagators: non-equilibrium correlation functions}

While the set of equations of motion (V.8) and (V.20) are reminiscent of those for the single particle wave functions and the single particle density matrix, in fact there is more information in the "effective" free field theory description afforded by the operator equations (V.8) and (V.20) combined with the field expansion (V.4). In particular, inserting the solution of the equations of motion (V.11 V.12 (and the similar ones for the antiparticles) into the expansion (V.4) for the field operators allow us to obtain any correlation function in the free field theory at equal or different times. These are the building blocks of any systematic perturbative expansion of processes of weak interactions. In particular the Feynman propagators, which are an essential ingredient in any calculation that involves neutrinos are given by

$$
\mathcal{S}_{\left(\alpha, \alpha^{\prime}\right)}^{F}\left(\vec{x}-\overrightarrow{x^{\prime}} ; t, t^{\prime}\right)=-i \int \frac{d^{3} k}{(2 \pi)^{3}} e^{i \vec{k} \cdot\left(\vec{x}-\vec{x}^{\prime}\right)} \quad\left[\left\langle\nu_{(\alpha)}(\vec{k}, t) \bar{\nu}_{\left(\alpha^{\prime}\right)}\left(\vec{k}, t^{\prime}\right)\right\rangle \Theta\left(t-t^{\prime}\right)-\left\langle\bar{\nu}_{\left(\alpha^{\prime}\right)}\left(\vec{k}, t^{\prime}\right) \nu_{(\alpha)}(\vec{k}, t)\right\rangle \Theta\left(t^{\prime}-t\right)\right]
$$

where the expectation values are in the initial density matrix, which is taken to be diagonal in the flavor basis in the present discussion.

The correlation (Wightmann) functions that enter in the Feynman propagator are found by using the leading order expansion (V.4 with the time evolution of the creation and annihilation operators given by eqns. (V.11V.12 and similar ones for $\beta_{\vec{k}, \lambda}^{(\alpha)}(t)$. With the purpose of highlighting the fast and slow time scales in the propagators, it is convenient to introduce the following functions that evolve on the slow time scale

$$
\begin{aligned}
& f_{k}(t)=\cos [\Omega(k) t]-i \cos (2 \theta) \sin [\Omega(k) t] \\
& g_{k}(t)=i \sin (2 \theta) \sin [\Omega(k) t]
\end{aligned}
$$

in terms of which the Heisenberg creation and annihilation operators of flavor states are written as follows

$$
\begin{aligned}
& \alpha_{\vec{k}, \lambda}^{(e)}(t)=e^{-i \bar{E}(k) t}\left[\alpha_{\vec{k}, \lambda}^{(e)}(0) f_{k}(t)+\alpha_{\vec{k}, \lambda}^{(\mu)}(0) g_{k}(t)\right] \\
& \alpha_{\vec{k}, \lambda}^{(\mu)}(t)=e^{-i \bar{E}(k) t}\left[\alpha_{\vec{k}, \lambda}^{(\mu)}(0) f_{k}^{*}(t)+\alpha_{\vec{k}, \lambda}^{(e)}(0) g_{k}(t)\right]
\end{aligned}
$$

and similarly for the antiparticle Heisenberg operators $\beta_{\vec{k}, \lambda}^{(\alpha)}(t)$.

A straighforward calculation of the Wightman functions yields the following results 


$$
\begin{aligned}
\left\langle\nu_{(e)}(\vec{k}, t) \bar{\nu}_{(e)}\left(\vec{k}, t^{\prime}\right)\right\rangle= & \left(\frac{\not k+\bar{M}}{2 \bar{E}(k)}\right) e^{-i \bar{E}(k)\left(t-t^{\prime}\right)}\left[\left(1-n^{(e)}(k)\right) f_{k}(t) f_{k}^{*}\left(t^{\prime}\right)+\left(1-n^{(\mu)}(k)\right) g_{k}(t) g_{k}^{*}\left(t^{\prime}\right)\right]+ \\
& \left(\gamma^{0} \frac{\not k-\bar{M}}{2 \bar{E}(k)} \gamma^{0}\right) e^{i \bar{E}(k)\left(t-t^{\prime}\right)}\left[\bar{n}^{(e)}(k) f_{k}^{*}(t) f_{k}\left(t^{\prime}\right)+\bar{n}^{(\mu)}(k) g_{k}^{*}(t) g_{k}\left(t^{\prime}\right)\right] \\
\left\langle\bar{\nu}_{(e)}\left(\vec{k}, t^{\prime}\right) \nu_{(e)}(\vec{k}, t)\right\rangle= & \left(\frac{\not k+\bar{M}}{2 \bar{E}(k)}\right) e^{-i \bar{E}(k)\left(t-t^{\prime}\right)}\left[n^{(e)}(k) f_{k}(t) f_{k}^{*}\left(t^{\prime}\right)+n^{(\mu)}(k) g_{k}(t) g_{k}^{*}\left(t^{\prime}\right)\right]+ \\
& \left(\gamma^{0} \frac{\not k-\bar{M}}{2 \bar{E}(k)} \gamma^{0}\right) e^{i \bar{E}(k)\left(t-t^{\prime}\right)}\left[\left(1-\bar{n}^{(e)}(k)\right) f_{k}^{*}(t) f_{k}\left(t^{\prime}\right)+\left(1-\bar{n}^{(\mu)}(k)\right) g_{k}^{*}(t) g_{k}\left(t^{\prime}\right)\right]
\end{aligned}
$$

where

$$
\not k \equiv \gamma^{0} \bar{E}(k)-\vec{\gamma} \cdot \vec{k}
$$

The Wightman function for the muon neutrino is obtained from that of the electron by the replacement $n^{(e)}(k), \bar{n}^{(e)}(k) \rightarrow n^{(\mu)}(k), \bar{n}^{(\mu)}(k)$, and $f_{k} \longleftrightarrow f_{k}^{*}$. The off-diagonal Wightman functions are given by

$$
\begin{aligned}
\left\langle\nu_{(\mu)}(\vec{k}, t) \bar{\nu}_{(e)}\left(\vec{k}, t^{\prime}\right)\right\rangle= & \left(\frac{\not k+\bar{M}}{2 \bar{E}(k)}\right) e^{-i \bar{E}(k)\left(t-t^{\prime}\right)}\left[\left(1-n^{(\mu)}(k)\right) f_{k}^{*}(t) g_{k}^{*}\left(t^{\prime}\right)+\left(1-n^{(e)}(k)\right) f_{k}^{*}\left(t^{\prime}\right) g_{k}(t)\right]+ \\
& \left(\gamma^{0} \frac{\not k-\bar{M}}{2 \bar{E}(k)} \gamma^{0}\right) e^{i \bar{E}(k)\left(t-t^{\prime}\right)}\left[\bar{n}^{(\mu)}(k) g_{k}\left(t^{\prime}\right) f_{k}(t)+\bar{n}^{(e)}(k) g_{k}^{*}(t) f_{k}\left(t^{\prime}\right)\right] \\
\left\langle\bar{\nu}_{(e)}\left(\vec{k}, t^{\prime}\right) \nu_{(\mu)}(\vec{k}, t)\right\rangle= & \left(\frac{\not k+\bar{M}}{2 \bar{E}(k)}\right) e^{-i \bar{E}(k)\left(t-t^{\prime}\right)}\left[n^{(\mu)}(k) f_{k}^{*}(t) g_{k}^{*}\left(t^{\prime}\right)+n^{(e)}(k) f_{k}^{*}\left(t^{\prime}\right) g_{k}(t)\right]+ \\
& \left(\gamma^{0} \frac{\not k-\bar{M}}{2 \bar{E}(k)} \gamma^{0}\right) e^{i \bar{E}(k)\left(t-t^{\prime}\right)}\left[\left(1-\bar{n}^{(\mu)}(k)\right) g_{k}\left(t^{\prime}\right) f_{k}(t)+\left(1-\bar{n}^{(e)}(k)\right) g_{k}^{*}(t) f_{k}\left(t^{\prime}\right)\right]
\end{aligned}
$$

the other off-diagonal Wightmann function is obtained from the one above by replacing $n^{(e)} \longleftrightarrow n^{(\mu)}$ and $f_{k} \longleftrightarrow f_{k}^{*}$.

We have specifically separated the "fast" evolution, encoded in the exponentials $e^{i \pm \bar{E}(k)\left(t-t^{\prime}\right)}$ and the "slow" evolution encoded in the functions $f_{k} ; g_{k}$ which oscillate with the small frequency $\Omega(k) \sim \Delta M^{2} / 2 \bar{E}(k)$. We emphasize that the propagators above are functions not only of the difference $\left(t-t^{\prime}\right)$ but also of the sum $\left(t+t^{\prime}\right)$ which reveals a truly non-equilibrium evolution. The manifest lack of time translational invariance reflects the fact that the density matrix which is diagonal in the flavor representation does not commute with the time evolution operator.

The discussion at the beginning of this section points out that these propagators are valid on time scales $t, t^{\prime} \gg 1 / \bar{M}$, for which the corrections arising from the interference between particle and antiparticle can be neglected. Therefore the correlation functions obtained from the effective field theory must be understood as being averaged over the fast time scales and their validity is restricted to slow time scales.

The free field theory propagators obtained above provide the main ingredients to carry out a study of the weak interactions in a neutrino background in a loop expansion.

\section{CONCLUSIONS AND DISCUSSIONS:}

Our focus was to study the evolution of a dense and or hot gas of flavor neutrinos as a consequence of oscillations and mixing. The goal was to establish an understanding of the dynamics directly from the underlying quantum field theory, beginning with the simplest case of free field theory and restricted to the two flavor case.

Such study leads to a deeper understanding of the various approximations invoked in the literature as well as recognizing the potential corrections. Even at the level of free field theory, which must be the starting point of any program to study the physics of oscillations and mixing in the weak interactions, this study reveals a wealth of dynamical phenomena that has not been explored before within the context of neutrino oscillations in a medium with neutrinos at finite density and temperature.

The most salient aspects of our study are the following:

- A hierarchy of time scales emerges associated with different interference phenomena. Oscillations on fast time scales $t<1 / \bar{M}$ are associated with the interference between particles and antiparticles while oscillations on 
slow time scales $t>\bar{M} / \Delta M^{2}$ arise from the interference between particle (or antiparticle) states with different masses. Observationally the situation for two flavors is that of near degeneracy, which entails that these time scales are widely separated. Furthermore in the relativistic limit with typical energy $\bar{E} \gg M_{1}, M_{2}$ there is an even shorter time scale $t \sim 1 / \bar{E}$.

- The terms that oscillate on fast scales feature coefficients that are determined by the overlap of positive and negative frequency wave functions of different masses. In the relativistic limit or in the case of near degeneracy as suggested by the recent observations, these terms are of order $\left(\Delta M^{2} / \bar{M}^{2}\right)^{2} \sim 10^{-6}$ (or smaller in the relativistic case), while the coefficients of terms that oscillate on the slow scales are of $\mathcal{O}(1)$ in terms of this ratio. During the short time scales both contributions are comparable, but for $t>>1 / \bar{M}$ the contribution from the overlap between particle and antiparticle states becomes subdominant being at least a factor $\left(\Delta M^{2} / \bar{M}^{2}\right)^{2} \sim 10^{-6}$ smaller than the oscillations on the slow time scale. For the values of $\bar{M}$ consistent with the recent bounds 46 ] the scale for fast oscillations is $\sim 10^{-15} s$ these are clearly too fast for relevant processes during BBN or neutrino processes in astrophysics, but may be relevant for early universe cosmology. Of course this possibility requires further and deeper studies.

- An initial flavor asymmetry relaxes to equilibrium via dephasing between modes that are not Pauli blocked with a power law $1 / t$ on slow time scales $t>k_{F} / \Delta m^{2}$ in the relativistic case $k_{F}>>\bar{M}$. We have obtained exact as well as approximate expressions for the time evolution of the distribution functions and off diagonal densities and discussed their asymptotic behavior, all of which display Pauli blocking between different flavors (see eqns. (IV.12 IV.15). For completeness we have also studied the case of an equilibrated gas of mass eigenstates which describes a situation of equilibrium in absence of interactions. The non-equilibrium oscillation dynamics leads to the production of particle-antiparticle pairs of flavored neutrinos with typical momenta $k \sim \bar{M}$. Since this phenomenon is a direct consequence of the overlap between particle and antiparticle states the pair yield is suppressed by the factor $\left(\Delta M^{2} / \bar{M}^{2}\right)^{2}$.

- The wide separation between the different time scales allows to describe the dynamics on the longer time scales in terms of an "effective" theory. In this effective description the Heisenberg creation and annihilation field operators for flavor neutrinos and antineutrinos obey the familiar Bloch type equations and the spinor structure is common to both flavors as well as the mass eigenstates. This effective description allows to obtain in a simple manner the dynamics of the distribution functions, off diagonal correlation functions and the non-equilibrium propagators, all of which must be understood as an average over the fast time scales and valid only on the slow scales.

While we have focused on the evolution of a gas of flavor neutrinos as an initial value problem we have not discussed how the initial state is "prepared". This is an important aspect of the physics of neutrino mixing and the weak interactions, since weak interactions only produce flavor states the initial state (or density matrix) must be "prepared" by weak interaction processes that occur on time scales much shorter than those in which such state will relax either via collisions or by oscillations. Clearly we have nothing to say yet on this aspect which deserves a thorough study.

Another aspect that deserves attention is that of the corrections to the "effective" theory described above. These corrections entail powers of the ratios that are small either in the nearly degenerate case or in the relativistic limit. In perturbation theory in the weak interactions, these "small" corrections could conceivably be comparable to perturbative corrections in $G_{F}$ the Fermi coupling, in which case the terms neglected in the effective theory must be kept on the same footing as the contributions in the weak coupling in the perturbative expansion. Clearly such possibility must be evaluated for the particular situation under consideration.

While we have focused on the dynamics in free field theory, the results will likely be valid in the interacting case in the case of a low density neutrino gas (or low temperatures). Under these circumstances the corrections to the evolution equations associated with forward scattering off the neutrino background (mean field), which is of order $G_{F}$ would be much smaller than $\Delta M^{2} / \bar{M}$ and the free field theory results for the evolution of the asymmetry may very well be valid. Furthermore, the weak interactions only affect the left handed neutrinos but not the right handed neutrinos which will oscillate as in a free field theory. The mass term will then entangle the oscillations of the right and left handed components. Such a process will be suppressed in the relativistic limit but may introduce yet another scale. The intriguing phenomenon of flavor pair production, a many body feature intrinsic to the field theory of neutrino mixing and oscillations. While it is not clear to the authors whether such phenomenon could have potential bearing in cosmology and astrophysics, it certainly is part of the fundamental aspects of neutrino mixing and oscillations and deserves further study.

We are currently studying these and other possible scenarios including interactions.

Having understood the regime of validity of the effective "long time" theory as well as having obtained the necessary non-equilibrium propagators we expect to address the issue of the propagation of neutrinos in a dense and or hot 
medium, including a neutrino background including not only forward scattering but also collisional processes 4, 6, 24, 25, 26] by implementing the methods of non-equilibrium quantum field theory [4]].

\section{Acknowledgments}

D.B. thanks the National Science Foundation for support under grant award PHY-0242134. He also thanks S. Reddy, A. Leibovich, V. Paolone, D. Naples for enjoyable and illuminating conversations.

[1] C. W. Kim and A. Pevsner, Neutrinos in Physics and Astrophysics, (Harwood Academic Publishers, USA, 1993).

[2] R. N. Mohapatra and P. B. Pal, Massive Neutrinos in Physics and Astrophysics, (World Scientific, Singapore, 1998).

[3] M. Fukugita and T. Yanagida, Physics of Neutrinos and Applications to Astrophysics, (Springer-Verlag Berlin Heidelberg 2003).

[4] G. G. Raffelt, Stars as Laboratories for Fundamental Physics, (The University of Chicago Press, Chicago, 1996).

[5] T. K. Kuo and J. Pantaleone, Rev. of Mod. Phys. 61, 937 (89).

[6] A. D. Dolgov, Surveys High Energ.Phys. 17, 91, (2002); Phys.Rept. 370, 333 (2002); Nuovo Cim. 117 B, 1081, (2003); hep-ph/0109155 ( talk at XV Rencontres de Physique de La Vallee d'Aoste, March, 2001); A.D. Dolgov, S.H. Hansen, S. Pastor, S.T. Petcov, G.G. Raffelt, D.V. Semikoz, Nucl.Phys. B632 (2002) 363-382.

[7] B. Pontecorvo, Zh. Eksp. Toer. Fiz. 34,247 [Sov. Phys. JETP 7,172 (1958)]; Zh. Eksp. Toer. Fiz. 53, 1717 [Sov. Phys. JETP 26, 984 (1968)]; Z. Maki, M. Nakazawa and S. Sakata, Prog. Theor. Phys. 28, 870 (1962).

[8] S. M. Bilenky and B. Pontecorvo, Phys. Rept. 41, 225 (1978).

[9] C. Giunti and M. Lavede, hep-ph/0310238 S.M. Bilenky, C. Giunti, J.A. Grifols, E. Masso, Phys.Rept. 379, 69 (2003); C. Giunti, Found. Phys. Lett. 17, 103 (2004); S. M. Bilenky and C. Giunti, Int. J. Mod. Phys. A16, 3931 (2001).

[10] A. Yu. Smirnov, hep-ph/0311259 hep-ph/0306075; hep-ph/0305106 hep-ph/0305106

[11] S. M. Bilenky, hep-ph/0402153 W.M. Alberico, S.M. Bilenky, hep-ph/0306239 S. M. Bilenky, hep-ph/0210128

[12] W. C. Haxton, nucl-th/9901076, Wick C. Haxton, Barry R. Holstein, Am.J.Phys. 68, 15 (2000).

[13] W. Grimus, hep-ph/0307149

[14] E. Roulet, astro-ph/0011570

[15] M. Beuthe, Phys.Rept. 375, 105 (2003).

[16] L. Wolfenstein, Phys. Rev. D17, 2369 (1978); Phys. Rev. D20, 2634 (1979); Phys. Lett. B194 197 (1987).

[17] S. P. Mikheyev and A. Yu Smirnov, , Yad. Fiz.42, 1441 (1985) (Sov. J. Nucl. Phys. 42, 913 (1985)); Nuovo Cimento C9, 17 (1986); Zh. Eksp. Toer. Fiz. 91, 7 (1986) (Sov. Phys. JETP 64, 4 (1986)).

[18] D. P. Kirilova and M. V. Chizhov, Phys.Rev. D58 073004 (1998); Phys.Lett. B393 375 (1997); hep-ph/9704269 Nucl.Phys. B534 447 (1998); Nucl.Phys.Proc.Suppl. 100360 (2001).

[19] M. Prakash, J. M. Lattimer, R. F. Sawyer, R. R. Volkas, Ann.Rev.Nucl.Part.Sci. 51, 295 (2001); S. Reddy, M. Prakash, J. M. Lattimer, Phys.Rev. D58, 013009 (1998)

[20] M. Prakash, J.M. Lattimer, J.A. Pons, A.W. Steiner, S. Reddy, Lect.Notes Phys. 578, 364 (2001), S. Reddy, M. Prakash, nucl-th/9508009

[21] D. G. Yakovlev, A. D. Kaminker, O. Y. Gnedin, P. Haensel, Phys.Rept. 354, 1 (2001).

[22] A. Dolgov, Sov. J. Nucl. Phys. 33,700 (1981); R. A. Harris and L. Stodolsky, Phys. Lett. 116B, 464 (1982); L. Stodolsky, Phys. Rev. D36, 2273 (1987).

[23] P. Mannheim, Phys. Rev. D37, 1935 (1988).

[24] G. Raffelt, G. Sigl and L. Stodolsky, Phys. Rev. D45, 1782 (1992); Phys. Rev. Lett. 70, 2363 (1993); G. Sigl and G. Raffelt, Nucl. Phys. B 406, 423 (1993).

[25] K. Enqvist, K. Kainulainen and J. Maalampi, Nucl. Phys. B349, 754, (1991).

[26] B. H. J. McKellar and M. J. Thompson, Phys. Rev. D49, 2710 (1994).

[27] R. Barbieri and A. Dolgov, Nucl. Phys. B 349, 743 (1991).

[28] V. A. Kostelecky, J. Pantaleone and S. Samuel, Phys. Lett. B315, 46 (1993); V. Kostelecky and S. Samuel, Phys. Lett. B318, 127 (1993); V. Kostelecky and S. Samuel, Phys. Rev. D49, 1740 (1994); V. Kostelecky and S. Samuel, Phys. Rev. D52, 3184 (1995).

[29] C. Lunardini and A. Y. Smirnov, Phys. Rev. D64, 073006 (2001).

[30] Y. Y. Y. Wong, Phys. Rev. D66, 025015 (2002); AIP Conf.Proc. 655, 240 (2003).

[31] K. N. Abazajian, J. F. Beacom and N. F. Bell, Phys. Rev. D 66, 013008 (2002).

[32] J. Pantaleone, Phys. Lett. 342, 250 (1995); J. Pantaleone, Phys.Rev.D46, 510 (1992) .

[33] Y. Z. Qian and G. M. Fuller, Phys. Rev. D51, 1479 (1995).

[34] G.Sigl, Phys.Rev. D51, 4035 (1995).

[35] N. F. Bell, R. R. Volkas, Y. Y. Y. Wong, Phys. Rev. D59, 113001 (1999), N. F. Bell, R. Foot, R. R. Volkas, Phys.Rev. D58, 105010 (1998); N. F. Bell, hep-ph/0311283

[36] D. Notzold and G. Raffelt, Nucl. Phys. B307, 924 (1988).

[37] J. F. Nieves, Phys. Rev. D40, 866 (1989). 
[38] J. C. D'Olivo and J. F. Nieves, Int. Jour. of Mod. Phys. A11, 141 (1996).

[39] A. Friedland and C. Lunardini, Phys. Rev. 68, 013007 (2003).

[40] M. Blasone and G. Vitiello, Phys.Rev. D60, 111302 (1999); M. Blasone, G. Vitiello, Annals of Physics 244,283 (1995); Erratum-ibid. 249, 363 (1996); E. Alfinito, M. Blasone, A. Iorio, G. Vitiello, Phys.Lett. B362, 91 (1995); E.Alfinito, M.Blasone, A.Iorio, G.Vitiello, Acta Phys.Polon. B27, 1493 (1996); M.Blasone, P.A.Henning, G.Vitiello, hep-ph/9605335 M. Blasone, P. P. Pacheco, H. W. C. Tseung, Phys.Rev. D67073011(2003), and references therein.

[41] K. Fujii, C. Habe and T. Yabuki, Phys. Rev. D64, 013011 (2001), Phys. Rev. D59, 113003 (1999); K. Fujii and T. Shimomura, hep-ph/0402274

[42] C-R. Ji and Y. Mischchenko, Phys. Rev. D65, 096015 (2002); hep-ph/0403073

[43] C. Giunti, hep-ph/0312256 hep-ph/0402217 J. H. Fried, hep-ph/0301231

[44] D. Boyanovsky, I.D. Lawrie, D.S. Lee, Phys.Rev. D54, 4013 (1996); S. M. Alamoudi, D. Boyanovsky, H. J. de Vega, R. Holman, Phys.Rev. D59, 025003 (1999); D. Boyanovsky, H.J. de Vega, S.-Y. Wang, Phys.Rev. D61, 065006 (2000).

[45] K. Eguchi et.al. (KamLAND collaboration), Phys. Rev. Lett.90, 021802 (2003).

[46] D. N. Spergel et. al. (WMAP collaboration), Astrophys.J.Suppl. 148 , 175 (2003).

[47] D. Boyanovsky, M. D'Attanasio, H.J. de Vega, R. Holman,Phys.Rev. D54 1748 (1996); D. Boyanovsky, M. D'attanasio, H.J. de Vega, R. Holman, Phys.Rev. D526805 (1995); D. Boyanovsky, M. D'Attanasio, H. J. de Vega and R. Holman, in Third Paris Cosmology Colloquium, Eds. H. J. de Vega and N. Sanchez, (World Scientific, Singapore, 1996); D. Boyanovsky, H. J. de Vega and R. Holman, in International School of Astrophysics D. Chanlonge, 5th course: Current topics in Astrofundamentel Physics, Eds. N. Sanchez and A. Zichichi, (World Scientific, Singapore, 1997). 